\title{
Measuring surface diffusion from nucleation island densities
}

\author{
Harald Brune, G. Steven Bales, ${ }^{*}$ Joachim Jacobsen, ${ }^{\dagger}$ Corrado Boragno, ${ }^{\ddagger}$ and Klaus Kern \\ Institut de Physique Expérimentale, Ecole Polytechnique Fédérale de Lausanne, CH-1015 Lausanne, Switzerland
}

(Received 15 June 1998; revised manuscript received 4 February 1999)

\begin{abstract}
We present a critical view of the analysis of experimental island densities acquired as a function of temperature in terms of barriers and prefactors for tracer diffusion at surfaces. We investigate the achievable precision for methods ranging from simple application of scaling laws, via integration of mean-field rate equations within various approximations for the capture rates, to kinetic Monte Carlo simulations that account for the various island shapes realized on square and hexagonal lattices. The discussion of theoretical models will be accompanied by variable temperature STM data for the nucleation of Ag on a Pt(111) surface. We introduce experimental methods to test for dimer diffusion and dissociation, as well as for transient mobility of monomers. Density scaling is analyzed in the presence of post-deposition mobility and easy adatom attachment to islands and other monomers. From extended kinetic Monte Carlo simulations we establish density scaling for the various island shapes on square and hexagonal lattices for coverages up to percolation, which is particularly relevant for methods working in reciprocal space. [S0163-1829(99)01231-X]
\end{abstract}

\section{INTRODUCTION}

Surface migration of single adatoms is one of the most fundamental processes in epitaxial growth and heterogeneous catalysis. In molecular-beam epitaxy it determines whether there is nucleation of islands on substrate terraces or step flow growth. In heterogeneous catalysis, surface diffusion of reactants often is the rate limiting process. Predictions on the growth mode and understanding of growth manipulation, e.g., by surfactants, as well as a fundamental understanding of the kinetics of surface chemical reactions, necessitate quantification of the surface diffusion coefficient $D$. Precise experimental values of activation barriers for surface diffusion are also needed as benchmarks for theoretical calculations.

One distinguishes the collective diffusion coefficient (also Fickian or chemical diffusion coefficient) of an ensemble of mutually interacting particles from the tracer diffusion coefficient (also intrinsic diffusion coefficient) describing the mean square displacement of one isolated random walker per unit time. The first type of diffusion is commonly measured by the decay of concentration profiles or by looking at density fluctuations of a lattice gas caused by thermal motion of the interacting particles. ${ }^{1}$ We focus on the second type of diffusion. For refractory metals, tracer diffusion has traditionally been studied by means of field ion microscopy (FIM) where the migration of single atoms (or clusters), adsorbed on single-crystal facets of the FIM tip, can be traced at low temperatures. ${ }^{2-4}$ Tracer diffusion has also been followed by means of direct inspection with scanning tunneling microscopy (STM)..$^{5-9}$ When quantifying diffusion rates from direct STM inspection of diffusing particles, one has to worry about possible tip-sample interactions, which might yield apparent diffusion barriers that are systematically reduced. ${ }^{5,8,10}$ For systems with large barriers, the tip influence can be suppressed by going to large gap widths. ${ }^{9}$ However, for systems with small diffusion barriers in situ STM observations of diffusion might be perturbed, even by very small tip-sample interactions. Therefore, alternative methods to quantify diffusion are highly appreciated.

Vapor phase epitaxy is characterized by tracer diffusion, since for typical growth rates the density of diffusing monomers $n_{1}$ is rather small (for $D / F>10^{5}$, with $F$ being the deposition rate, $\left.n_{1} \ll 10^{-3} \mathrm{ML}\right)$. Therefore, an alternative way to quantify tracer diffusion is to measure island densities that form during deposition onto a single-crystal surface as a function of temperature and deposition rate. The activation energy $E_{m}$, and attempt frequency $\nu_{0}$, for surface diffusion are then commonly extracted by comparison of measured island densities with those predicted by mean-field nucleation theory. ${ }^{11,12}$ This nucleation method experienced a revival when densities for monolayer high, two-dimensional islands became accessible in real space by STM. ${ }^{13-15}$ Apart from diffusion studies on isotropic substrates, the nucleation method was applied to study the effect of (homogeneous) strain on surface diffusion, ${ }^{16}$ to explore diffusion on inhomogeneous substrates with dislocations, ${ }^{17-19}$ it even permits diffusion studies in the presence of point defects, such as surfactants. Extensions of STM to variable (low) temperatures $^{20-22}$ provide the basis on which to study any system in the irreversible growth regime where dimers are stable. These low temperature data can be analyzed in terms of a critical nucleus size $i=1$, for which the only free parameters

the barrier, $E_{m}$ and the attempt frequency, $\nu_{0}$ of surface diffusion.

Island densities are also accessible to integrating surface science techniques. They can be inferred for instance from diffraction scans under out of phase conditions in thermal helium atom scattering, ${ }^{23}$ and high-resolution low-energy electron diffraction. ${ }^{24}$ Since integrating techniques average over large areas, they facilitate acquisition of statistically significant numbers. However, step effects cannot be discerned, which is not a problem for samples with large terraces, but more importantly, assumptions on the island distance and size distributions enter into the interpretation of diffraction scans as number densities. ${ }^{23,24}$ The side bands in diffracted intensities reflect the most probable island distance, whereas 
the island density $n_{x}$ is correlated with the mean island distance. Moreover, a single diffraction scan only reports a onedimensional projection of island distances. A careful analysis of diffraction spot profiles, however, can yield the true island densities in real space. ${ }^{25,26}$ However, diffraction techniques require usually coverages above the onset of saturation, where coalescence becomes important. Coalescence effects on density scaling are quite different for various island shapes.

In the present paper, we discuss the measurement of the surface diffusion coefficient by direct comparison of experimental observations of low-temperature, irreversible island growth with scaling theory, kinetic Monte Carlo (KMC) simulations that account for the lattice and island symmetry, and results obtained from integrating mean-field rate equations within various approximations for the capture numbers. As an experimental example we will analyze $\mathrm{Ag} / \mathrm{Pt}(111)$. Emphasis will be placed on the precision to which $E_{m}$ and $\nu_{0}$ can be determined from experimental island densities upon applying the various theoretical calculations. Evidently, the precision can be increased in investigating the largest possible temperature window. To the high temperature end, one is limited by dimer instability and/or diffusion. Towards low temperatures $\left(D / F<10^{5}\right)$ there is no intrinsic limitation, apart from the analysis becoming complicated, since details such as attachment to islands, transient mobility of condensing atoms, and post deposition mobility begin to play a role. We will show how to test for these effects experimentally and how to account for them in the analysis. Since meanfield nucleation theory has difficulties in accounting for island correlations, it fails to give a quantitative description of coalescence (and island-size distributions). Coalescence effects are therefore studied by means of KMC for the relevant island shapes appearing in metal growth on isotropic substrates.

The outline of the paper is as follows. We first present various experimental methods to determine the critical cluster size $i$ and to test for dimer diffusion. Experimental saturation island densities for $\operatorname{Ag} / \operatorname{Pt}(111)$ (at $\theta=0.12 \mathrm{ML}$ ) are then analyzed in comparison with the scaling law from nucleation theory, self-consistent mean-field rate theory, mean-field rate theory applying various approximations for the capture rates, and KMC simulations. We show that a straightforward scaling analysis, in terms of slope and intercept of the Arrhenius plot of saturation island densities, is only valid if nucleation entirely takes place during deposition. This condition is fulfilled for $D / F>10^{5}$, which limits the precision to which migration barriers can be extracted from island densities by application of scaling laws. For $D / F<10^{5}$ we find strong deviations from scaling due to post-deposition mobility of monomers. Rate equations and KMC simulations permit the analysis of island densities also in this regime. For our experimental example of $\mathrm{Ag} / \mathrm{Pt}(111)$ this enables one to address a range of $D / F$ of 10 orders of magnitude, which significantly increases the precision to which $E_{m}$ and $\nu_{0}$ can be determined. Then, the effect of island shapes is addressed by comparing $\mathrm{KMC}$ simulations on square and hexagonal lattices performed with various choices for diffusion of adatoms around the island edges. With these simulations we investigate coalescence and quantify density scaling for diffusion limited aggregation (DLA), dendritic, and compact islands and coverages up to the percolation threshold.

\section{CRITICAL CLUSTER SIZE}

Classical mean-field nucleation theory ${ }^{11,27-29}$ gives the following expression for the saturated number density of stable islands $n_{x}$ for the case of complete condensation and two-dimensional (2D) islands:

$$
n_{x} \simeq \eta(\theta, i)\left(\frac{D}{F}\right)^{-\chi} \exp \left[\frac{E_{i}}{(i+2) k T}\right]
$$

where the scaling exponent $\chi=i /(i+2) i$ denotes the critical cluster size, and $E_{i}$ its binding energy $\left(E_{1}=0\right)$. Critical clusters turn into stable ones upon incorporation of one extra atom. Stable refers to growing more rapidly than decaying in the course of deposition. Accordingly, in thermodynamic terms, the critical cluster size $i$ is where the Gibbs free energy as a function of cluster size has its maximum. In nucleation theory, $i$ has been obtained self-consistently through minimization of $n_{x}$ within a pair binding model for $E_{i} \cdot{ }^{12}$

The application of Eq. (1) for the measurement of activation barriers requires a range of growth conditions in which the critical size remains constant. In practice, this only occurs for low temperatures when $i$, which depends on the surface symmetry, is small. ${ }^{30-32}$ Values of $i=1$ or 2 are expected for hexagonal surfaces and $i=1,3$, or 8 (Ref. 33) for surfaces with square symmetry. For larger temperatures the nonequilibrium critical size changes continuously with growth conditions (including coverage) and the utility of Eq. (1) is diminished.

Measuring $D$ is most convenient when dimers are stable so that $i=1$ and Eq. (1) reduces to $n_{x}=\eta(\theta, 1)(D / F)^{-1 / 3}$. Without additional fit parameters, as, e.g., cluster binding energies $E_{i}$, this gives direct access to $E_{m}$ and $\nu_{0}$ for surface migration of single adatoms via the slope and intercept of a line fit to the experimental island densities represented in an Arrhenius plot. We will analyze below the accuracy and the extent to which this approach is applicable (see Sec. III). For example, there are corrections to the precise value of the exponent $\chi$ when the islands are ramified ${ }^{34}$ and a small logarithmic correction to the dependence on $D / F$ is needed to correctly account for the adatom collision rate. ${ }^{11,29}$ However, before proceeding, one must first verify experimentally in which temperature and flux range the dimers are stable. In the current section various ways to test whether $i=1$ are discussed.

\section{A. Dimer stability}

A direct way to test whether $i=1$ is to measure the temperature threshold for the onset of Ostwald ripening for a population of very small islands containing mostly dimers. A high abundance of such small islands is created by deposition of $\sim 0.1 \mathrm{ML}$ at low temperature such that $D / F<10^{3}$. Under these conditions, monomers reach each other only after deposition leading to a high density of small islands, mostly dimers and trimers as well as very few larger islands. The same result is obtained when depositing at temperatures where diffusion is entirely frozen, followed by a gentle an- 


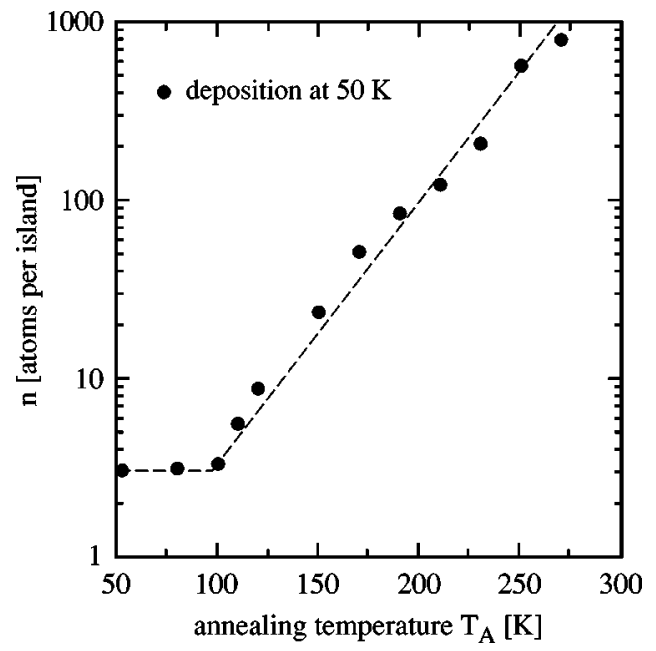

FIG. 1. Investigation of dimer stability and 2D Ostwald ripening for $\mathrm{Ag} / \mathrm{Pt}(111)$ through observation of the mean island size $n$ with annealing temperature by means of STM. The island size stays constant until it reveals an exponential increase at $T>100 \mathrm{~K}$, the onset of which is attributed to dimer dissociation. The initial distribution of small islands was produced by deposition of $0.1 \mathrm{ML} \mathrm{Ag}$ onto $\mathrm{Pt}(111)$ at $50 \mathrm{~K}$.

nealing to activate diffusion. The mean island size expected from integrating rate equations for both cases is about 3 atoms, ${ }^{35}$ in agreement with experiments on square ${ }^{35}$ and hexagonal lattices [see Fig. 1 for $\mathrm{Ag} / \mathrm{Pt}(111)]$. Although the mean island size is three, the population contains a substantial fraction $(\sim 40 \%)$ of dimers-the stability of which is the subject of our interest.

To investigate the dimer stability and to derive estimates on its dissociation barrier $E_{\text {diss }}$, the density $n_{x}$ of these small islands, respectively, their average size $n=\theta / n_{x}$, is monitored by STM as a function of annealing temperature. In the experiment represented in Fig. 1 the surface was annealed for thirty minutes and than imaged before annealing at the next, higher temperature. One generally observes that the average island size $n$ stays constant, until it suddenly increases at a well-defined threshold temperature. In the temperature regime of constant $n$ the most fragile objects in the population, namely the dimers, must neither dissociate nor diffuse. The increase in island size upon annealing beyond the threshold temperature marks the onset of dimer diffusion and/or dissociation. In the course of further annealing also islands larger than dimers become unstable. This leads to coarsening due to more rapid diffusion and/or dissociation of smaller islands that hence disappear to the expense of large ones. The coarsening is called 2D Ostwald ripening if dissociation is its cause.

In Fig. 1 there is an abrupt transition from the regime of constant island size to the onset of Ostwald ripening located at $100 \mathrm{~K}$. From this threshold temperature and the employed annealing period an estimate of $E_{\text {diss }}=E_{m}+E_{b}=320$ $\pm 20 \mathrm{meV}$ for the barrier to dissociate a $\mathrm{Ag}$ dimer on $\mathrm{Pt}(111)$ can be inferred (under the assumption of equal prefactors for dissociation and diffusion). With $E_{m}=168$ $\pm 5 \mathrm{meV}$ (see below), the dimer bond energy evaluates to $E_{b}=150 \pm 20 \mathrm{meV}$. The value of $E_{\text {diss }}$ implies that on the much shorter time scale of deposition ( $100 \mathrm{~s}$ for $0.1 \mathrm{ML}$ ), the $\mathrm{Ag}$ dimer is stable and immobile up to $\sim 110 \mathrm{~K}$. Dimer in- stability should begin to affect island densities at $\sim 120 \mathrm{~K}$, where the lifetime of a dimer is only about one second.

\section{B. Dimer mobility}

In addition to the dimer stability issue, it is important for nucleation studies to know when and how dimer diffusion, or more generally, cluster diffusion affects island densities. This subject has been treated in Refs. 11, 29, 36 and 37. As mentioned above, the onset temperature for coarsening of small islands serves to exclude both, dimer instability and diffusion, and thus the temperature and flux regime of irreversible growth $(i=1)$ and immobile clusters is unambiguously identified. If such information is missing, however, one needs to know whether at all, and if yes, when dimer diffusion affects $i=1$ density scaling.

In the majority of systems investigated so far there was no influence of dimer diffusion and $i=1$ density scaling persisted until termination by a transition to $i \geqslant 2$ through dimer dissociation. ${ }^{37}$ Despite this observation, dimers may start to diffuse well below the threshold temperature for their dissociation. ${ }^{46,38}$ Therefore, dimer diffusion may well happen in the $i=1$ regime, however, without perturbing the scaling law Eq. (1). ${ }^{6,13}$ In simulations, on the other hand, one can produce a dimer diffusion regime with different scaling laws located between the $i=1$ and the $i=2$ regime. $^{36}$

There are two reasons why this intermediate regime is not observed in many experimental systems: (i) the energy barrier for dissociation of dimers is often close to that of dimer translation, and (ii) cluster dissociation has an intrinsically greater effect on island densities than cluster diffusion. One can estimate which conditions must be met for that dimer diffusion perturbs density scaling before dimer dissociation does, based on the following results that were derived from mean-field theory ${ }^{29}$ and that have been verified in simulations. ${ }^{37}$

(i) The scaling of island densities becomes only affected by dimer diffusion if $\nu_{2}>\nu_{\text {diss }} / n_{x}$, where $\nu_{2}$ is the dimer diffusion rate and $\nu_{\text {diss }}$ its dissociation rate.

(ii) The threshold temperature or flux $F$, where dimer diffusion starts to reduce the island density below the value caused by pure monomer migration is given by $\nu_{2}^{3} / \nu_{1}^{2} \gg F$, where $\nu_{1}$ denotes the monomer hopping rate.

Conditions (i) and (ii) must both be met in order for dimer diffusion to affect nucleation island densities. Since $n_{x}$ is typically of the order of $10^{-3}$ or smaller, (i) requires considerably smaller barriers for dimer diffusion than for dimer dissociation, $E_{d i s s}-E_{2}>3 \ln 10 k T$. At the same time, (ii) requires $E_{2}$ being rather close to $E_{m}, E_{2}<5 \ln 10 k T$ $+2 / 3 E_{m}$ for typical values of $F=10^{-3} \mathrm{~s}^{-1}$ and $\nu_{0}$ $=10^{12} \mathrm{~s}^{-1}$. (We assumed similar prefactors for all three processes.)

For $\mathrm{Ag} / \mathrm{Pt}(111)$ we have estimated $E_{\text {diss }}$ above, $E_{m}$ will be determined below, and for $E_{2}$ we can estimate from the annealing experiments that $E_{2} \geqslant E_{\text {diss }}$, therefore (i) and (ii) are not met and this system will have its $i=1$ regime terminated towards high temperatures by dimer dissociation. For distinguished systems such as $\mathrm{Pt} / \mathrm{Pt}(111)$, there are FIM results that quantify $\nu_{1}(T)$ and $\nu_{2}(T)$ thus enabling predictions on the temperature and flux range in which dimer diffusion starts to play a role. ${ }^{38}$ Typically, however, if one sets out to 
measure $E_{m}, E_{2}$ and $E_{\text {diss }}$ are also unknown, thus hampering estimates with conditions (i) and (ii). Therefore we briefly discuss general trends of dimer diffusion on square and hexagonal lattices that permit one to estimate $E_{2}$ vs $E_{m}$.

On surfaces with square symmetry monomer diffusion can take place by exchange. ${ }^{39,40}$ For such systems, dimer diffusion may have a lower activation energy than monomer diffusion, ${ }^{41,42}$ and if dimers are stable entities, their diffusion will largely influence island densities. On the other hand, for square surfaces with ordinary hopping diffusion, ${ }^{43}$ the barrier for dimer diffusion is significantly larger than for monomers, ${ }^{44}$ thus violating condition (ii). Consequently, nucleation experiments on square surfaces with hopping diffusion show a regular $i=1$ behavior without any signature of dimer diffusion. ${ }^{35,45}$

On hexagonal lattices, dimers may spin around their center with a barrier comparable to $E_{m} \cdot{ }^{46,47}$ However, since there is no net translation associated with this intracell rotation it does not affect density scaling. In contrast to this "easy" rotation, a center-of-mass translation of dimers is typically associated with much higher barriers than monomer diffusion, as indicated by experiment ${ }^{38,46}$ and theory. ${ }^{42,47}$

These general trends suggest that for metal-on-metal systems cluster diffusion does not affect density scaling square lattices with exchange diffusion are possible candidates for an exception. However, care has to be taken with these trends since often so-called chemical differences are good for surprises. Therefore, we emphasize the value of simple annealing experiments as described in the previous subsection to experimentally exclude dimer mobility.

\section{Scaling of island sizes}

The dimer annealing experiment discussed above (cf. Fig. 1) establishes the $i=1$ temperature range without employing nucleation or scaling theory. Therefore, it supplies information that serves to test predictions from these theories. One of the predictions of Eq. (1) is the flux dependence $n_{x} \propto F^{\chi}$ with $\chi=1 / 3$ for $i=1$. For $\mathrm{Ag} / \mathrm{Pt}(111)$ we find $\chi=0.32$ \pm 0.05 by a flux series performed at $90 \mathrm{~K}\left(4 \times 10^{-5} \leqslant F \leqslant 8\right.$ $\left.\times 10^{-3} \mathrm{ML} / \mathrm{s}\right)$. This result confirms the theoretical scaling exponent; however, the error margin is too large to experimentally pin down the precise value of $\chi$, which is needed for the precise analysis of $n_{x}(T)$ in terms of $D(T)$. The considerable error margin is due to the weak influence of the flux on island density often being close to statistical errors. Apart from on exception where $\chi$ could be determined by experiment with sufficient precision $(\chi=0.32 \pm 0.01$, see Ref. 35) precise quantification of the exponent in Eq. (1) is most conveniently done from kinetic Monte Carlo simulations $\mathrm{s}^{34,48,49}$ allowing for as much statistics as computer time permits (see below). Despite the experimental difficulties in acquiring the flux dependence of $n_{x}$, it bears valuable information as it enables one a clear distinction of the critical cluster sizes in the various regimes of $T$ and $F$ (see Ref. 35) and to determine the dimensionality of diffusion for the anisotropic case. $^{50,51}$

From scaling theories it is predicted that island-size distributions, when scaled properly, all fall onto common curves that only depend on $i^{30,32,51-54}$ Figure 2 shows these curves obtained empirically for $i=1,2$, and $3 .{ }^{32}$ The most

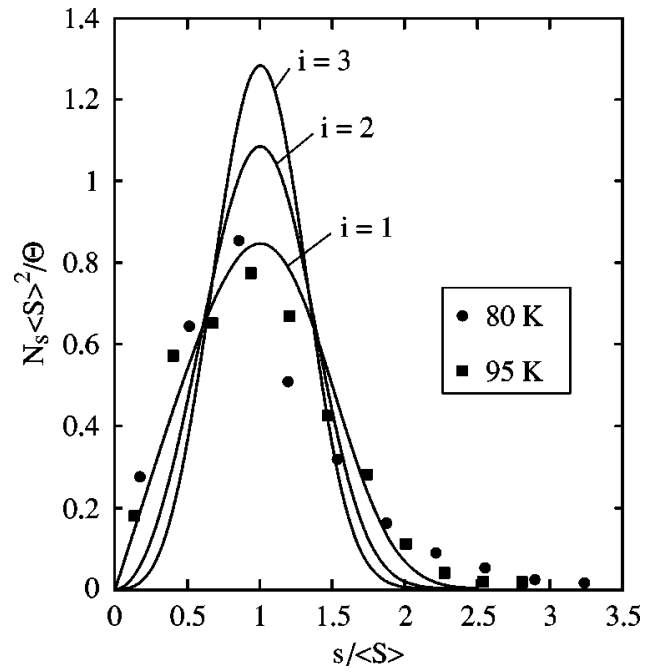

FIG. 2. Scaled island-size distributions extracted from a series of STM images obtained after deposition of $0.12 \mathrm{ML} \mathrm{Ag}$ onto $\mathrm{Pt}(111)$ at $80 \mathrm{~K}$ and $95 \mathrm{~K}$ in comparison with theoretical curves from Amar and Family (Ref. 32).

pronounced difference between the theoretical curves is the height of the maxima and the peak width. The former increases with $i$, the latter decreases due to conservation of the total area under the curve. The experimental island sizes obtained for $\mathrm{Ag} / \mathrm{Pt}(111)$ at 80 and $95 \mathrm{~K}$ fall into a common distribution that agrees well with the theoretical curve for $i$ $=1$. This is in accordance with the experimental result of dimers being stable at these temperatures derived above.

Island-size distributions are sensitive indicators for dimer and, in general, cluster mobility. ${ }^{37,55,56}$ They become significantly sharper through cluster motion. This change to the size distributions appears very early, already before dimer diffusion could alter the density. In that sense, the experimental size distributions in Fig. 2 also serve to exclude dimer mobility for $\mathrm{Ag} / \mathrm{Pt}(111)$ at 80 and $95 \mathrm{~K}$. They have a full widths at half maximum of 0.55 , which is the predicted value for ordinary $i=1$ nucleation without cluster motion, in agreement with the coarsening experiment described above.

\section{ANALYSIS OF ISLAND DENSITIES}

In the following, we discuss several methods to analyze temperature-dependent island densities $n_{x}(T)$ acquired in the $i=1$ regime in terms of $E_{m}$ and $\nu_{0}$ of surface diffusion. the discussion is in order of increasing precision, but also complexity.

\section{A. Scaling theory}

The simplest and most widely used approach is to apply Eq. (1) to the linear part in the Arrhenius plot of $n_{x}$, where dimers are stable $(i=1)$, and extract the migration barrier and the attempt frequency from the slope of the linear regression to the data and its intersection with the ordinate. As we will see in detail below, the $i=1$ regime obeys linear density scaling to a good approximation only down to $D / F=1$ $\times 10^{5}$. Towards lower temperatures, or larger deposition 


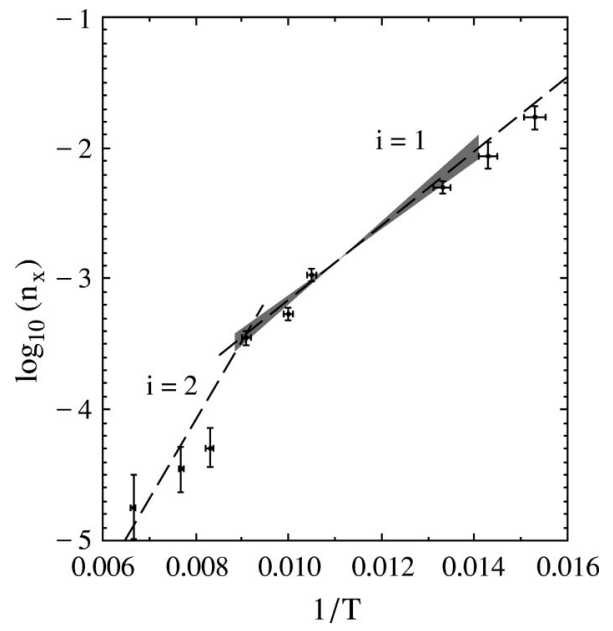

FIG. 3. Arrhenius plot of saturation island densities measured at $\theta=0.12 \mathrm{ML}$ for $\mathrm{Ag} / \mathrm{Pt}(111)$. A linear fit in that part of the for $i$ $=1$ regime, where Eq. (1) is a good approximation $\left(D / F \geqslant 10^{5}\right)$, yields a first estimate of $E_{m}$ and $\nu_{0}$. The dashed line for $i=2$ shows the slope expected from a dimer bond energy of $E_{b}=150$ $\pm 20 \mathrm{meV}$, as derived from the onset of dimer dissociation in Fig. 1.

rates, nucleation no longer entirely takes place during deposition, which is the condition under which Eq. (1) was derived.

Measured saturation island densities ( $\theta=0.12 \mathrm{ML})$ for our example of $\mathrm{Ag} / \mathrm{Pt}(111)$ are shown in Fig. 3. The Arrhenius plot shows two roughly linear regimes labeled $i=1$ and $i=2$. In agreement with the dimer bond energy inferred above from the onset of Ostwald ripening, the transition between the two is located at $110 \mathrm{~K}$. Taking the STM data in the part of the $i=1$ regime to which Eq. (1) can be applied, one obtains $E_{m}=170 \pm 15 \mathrm{meV}$ and $\nu_{0}=3 \times 10^{13.0 \pm 0.8} \mathrm{~s}^{-1}$ (see line fit and shaded error region in Fig. 3). To extract the attempt frequency from the intercept, the proportionality factor in Eq. (1) was set to $\eta(\theta=0.12 \mathrm{ML}, i=1)=0.25$; this value has been given by Venables (see curve for $i=1$ in Fig. 6c of Ref. 27); it is confirmed by our self-consistent analysis and KMC simulations-see below. Notice, however, that care must be taken when selecting the data attributed to the linear regime. From Fig. 3 it is not at all obvious that the last two data points at 70 and $65 \mathrm{~K}$ fall beyond the applicability range of Eq. (1). We obtained a slightly lower barrier of $E_{m}=157 \pm 10 \mathrm{meV}\left(\nu_{0}=5 \times 10^{13.0 \pm 0.7} \mathrm{~s}^{-1}\right)$ when we formerly analyzed the data down to $65 \mathrm{~K}$ with a linear fit $\left(D / F=2 \times 10^{3}\right)$ (see Ref. 15).

The dashed line for $i=2$ shows the expected slope from the above values for $E_{b}$ and $E_{m}$ upon application of Eq. (1). It describes well the island densities found for $T>110 \mathrm{~K}$. However, these densities are rather small and therefore subject to larger statistical and systematic errors. The latter are caused by the presence of steps which act as heterogeneous sinks for diffusing monomers competing with homogeneous nucleation on terraces. ${ }^{57-59}$

\section{B. Rate theory}

Another common approach by which to analyze experimental island densities is to integrate rate equations of nucle- ation within certain approximations for the capture numbers $\sigma{ }^{15,8}$ This method overcomes several restrictions of scaling theory. Rate theory enables one to account for postdeposition mobility and therefore to analyze experimental data also beyond $D / F=10^{5}$. The wider range of $D / F$ accessible, in particular its extension towards lower temperatures, considerably improves the precision with which parameters of diffusion can be extracted. Furthermore, analysis with rate theory opens up the investigation of densities taken at any coverage within and below the "saturation"' regime, whereas the scaling law expressed in Eq. (1) is strictly valid only for the maximum island densities attained during deposition. The coverage where this maximum is located can vary with $D / F$ and also with island shape. Therefore the scaling of densities acquired at fixed coverage can slightly differ from Eq. (1). In this subsection we will investigate this variation and the strength and weakness of several classical approximations for the capture numbers in comparison to selfconsistent solutions. ${ }^{34}$

The rate equations for monomers and stable islands read for the case of metal epitaxy at low temperatures, i.e., dimers being stable and immobile, no re-evaporation (complete condensation) and 2D islands [compare Eqs. (2.3), (2.5), (2.6), and (2.8) in Ref. 27]:

$$
\frac{d n_{1}}{d t}=F-2 \sigma_{1} D n_{1}^{2}-\sigma_{x} D n_{1} n_{x}-\kappa_{x} F\left(F t-n_{1}\right)-2 \kappa_{1} F n_{1}
$$

$$
\frac{d n_{x}}{d t}=\sigma_{1} D n_{1}^{2}+\kappa_{1} F n_{1}
$$

The terms on the right-hand side of Eq. (2) denote the increase of monomer density due to deposition with flux $F$, its decrease due to the encounter of two diffusing atoms under creation of a dimer - associated with the disappearance of two atoms, the decrease occurring when a monomer is captured by a stable island. The last two terms denote the decrease of $n_{1}$ caused by direct impingement onto $\left(\kappa_{1}=\kappa_{x}\right.$ $=1$ ), or into the immediate vicinity of (expressed by $\kappa_{1}$, $\kappa_{x}>1$ ), stable islands and monomers. For typical values of $\kappa$ these direct impingement terms are small with respect to the first three terms in Eq. (2). In Eq. (3) the terms on the righthand side account for the increase of stable island density $n_{x}$ due to creation of dimers, first when two monomers meet by diffusion, and second upon direct deposition onto an adatom. For sake of comparison with the self-consistent analysis above, coalescence is neglected in Eq. (3); incorporation would add a further term of the form $-2 n_{x}\left(F-d n_{1} / d t\right) .^{27}$

The capture numbers in Eqs. (2) and (3) describe the capability of islands or monomers to capture diffusing adatoms, i.e., they determine the island growth rate. They are proportional to the gradient of the monomer concentration $\partial n_{1}(\mathbf{r}, t) / \partial r$ at the island edge, to the island radius, and inversely proportional to the mean monomer density $n_{1}$ appearing in the rate equations. The capture numbers, therefore, generally imply the solution of a two-dimensional diffusion problem involving spatial correlations between islands. Approximate solutions to this problem were suggested and discussed long ago. ${ }^{11}$ There are basically two approximations for the diffusive loss of monomers towards islands. The first one assumes that the islands are placed on a 


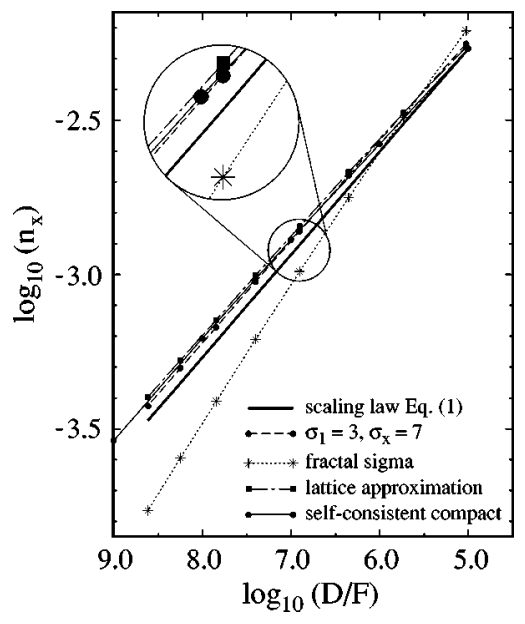

FIG. 4. Results from integrating rate Eqs. (2) and (3) within various approximations to the capture numbers $\sigma_{1}$ and $\sigma_{x}$ up to $\theta=0.12 \mathrm{ML}$. The approximations are compared to the scaling law Eq. (1) $(\eta=0.25, \chi=1 / 3)$ and to the self-consistent solutions for compact islands reported in Ref. 34. Fractal sigma stands for the geometric concept applied to fractal islands. In all cases coalescence has been neglected.

periodic lattice. Since islands nucleated on a homogeneous substrate are randomly distributed, this so-called lattice approximation ${ }^{11,61}$ overestimates island correlations and thus represents an upper bound to $\sigma$. The second approximation is the uniform depletion approximation representing a lower bound to $\sigma .{ }^{11}$ The uniform depletion approximation has been solved selfconsistently for all cluster sizes, and perfect agreement of the density scaling with KMC simulations has been demonstrated. ${ }^{34}$ These self-consistent solutions are the most accurate approximations of the capture numbers to date. Nevertheless, a comparison to the classical approximations is worthwhile since they significantly reduce the calculational effort.

In Fig. 4 and Table I, we compare the density scaling obtained with the various approximations for $\sigma$, to the scaling law Eq. (1) with $\chi=1 / 3$ and $\eta=0.25$ for a fixed coverage of $0.12 \mathrm{ML}$. We mention already at that point that there is a small island shape effect on the scaling exponent. We find from the KMC simulations to be discussed below that compact islands have a scaling exponent $\sim 5 \%$ below the classical one, whereas diffusion-limited aggregation (DLA) clus-

TABLE I. Prefactors $\eta$ and slopes $\chi$ in Eq. (1) for $\theta=0.12$ and $i=1$ obtained from linear regression to mean-field calculations within several approximations for $\sigma$ (see also data displayed in Fig. 4).

\begin{tabular}{lcc}
\hline \hline approximation & $\eta$ & $3 \times \chi$ \\
\hline Eq. (1) & 0.250 & 1.000 \\
Fractal sigma & $0.99 \pm 0.08$ & $1.30 \pm 0.01$ \\
$\sigma_{1}=3, \sigma_{x}=7$ & $0.25 \pm 0.01$ & $0.981 \pm 0.003$ \\
$\sigma_{1}=3, \sigma_{x}$ latt. appr. & $0.22 \pm 0.01$ & $0.951 \pm 0.006$ \\
Self-consistent compact & $0.27 \pm 0.02$ & $0.99 \pm 0.01$ \\
$\sigma_{1}$ from Ref. 34, & $0.30 \pm 0.01$ & $1.025 \pm 0.002$ \\
$\sigma_{x}$ lattice approximation & & \\
Self-consistent fractal & $0.27 \pm 0.01$ & $1.027 \pm 0.006$ \\
\hline \hline
\end{tabular}

ters have a by $\sim 2 \%$ larger scaling exponent. Therefore, the goal of the capture numbers is not to exactly reproduce the classical scaling exponent of $\frac{1}{3}$ but rather to give the correct exponents for the respective island shape.

One of the approaches to $\sigma$ that was pointed out in the early literature is the geometric concept. ${ }^{60}$ In this concept the $2 \mathrm{D}$ diffusion equation is not solved and capture rates are interpreted as capture cross sections and therefore set proportional to the island perimeter seen by the approaching monomers. When applied to fractal islands, this yields $\sigma_{x}=2$ $+x^{1 / 1.7}$, with $x$ being the island size in atoms; the constant of 2 accounts for atoms diffusing towards sites adjacent to the island perimeter. ${ }^{15}$ It is seen from Fig. 4 and Table I that the geometric concept is inconsistent, since it yields a significantly larger slope in the Arrhenius representation of the island densities than that expected from Eq. (1). The inconsistency comes from disregarding the diffusion field towards the islands, which is driven by the gradient in the monomer concentration.

An alternative approximation, being even simpler while yielding much better results, is to assume constant capture rates. ${ }^{61}$ This assumption is based on the fact that more sophisticated approximations for the capture rates show that these do not change very much in the coverage range close to saturation [see, e.g., Fig. 2(a) of Ref. 11]. Therefore, constant capture rates can be chosen such that they match Eq. (1) for a small range of saturation coverages. From Fig. 4 and Table I it is seen that $\sigma_{1}=3$ and $\sigma_{x}=7$ work very well for $\theta=0.12$ ML. The slope is by $2 \%$ below that produced by the classical scaling exponent. Also, the absolute number densities come out exactly as predicted from classical scaling theory (see $\eta$ value in Table I). However, constant capture rates do not give the correct coverage dependence of $n_{x}$, as the real capture rates vary considerably from the beginning of deposition up to saturation. The constants used above are only adequate for reproducing the density at 0.12 monolayers, since constant rate coefficients predict that the island density will increase as $\theta^{1 / 2}$ instead of saturating.

Approaches that work for all coverages up to saturation are those where the $2 \mathrm{D}$ diffusion equation is solved. Numerically integrating the rate equations within the lattice approximation yields a slope which is $5 \%$ below the classical exponent of $\chi=\frac{1}{3}$ (see Table I) and therefore well suited for compact islands. (For simplicity $\sigma_{1}$ was assumed to be constant, the value of $\sigma_{1}=3$ was chosen since $\sigma_{1}$ varies from 2 to 4 in the coverage range of $10^{-3}$ to $10^{-1} \mathrm{ML}^{11}$ ) Table I shows that the larger scaling exponent of fractal islands can be obtained in the lattice approximation when using the expression proposed by Bales and Chrzan for $\sigma_{1}{ }^{34}$

For the self-consistent solutions expressions have been derived for compact and fractal islands. ${ }^{34}$ The calculation for fractal islands is seen from Table I to yield the appropriate exponent, whereas the compact island exponent comes out slightly too large compared to our KMC results.

The comparison in Fig. 4 and Table I shows that the lattice approximation and the self-consistent solutions yield results that are consistent with each other within a small error margin of $\pm 1 \%$ for fractal islands, and of $\pm 2 \%$ for compact islands. Unknown island shape, as e.g., present in diffraction experiments, is a source of systematic errors of $\pm 4 \%$. The precision of absolute number densities, i.e., of $\eta$, is in the 
range of $\pm 10 \%$ enabling determination of $\nu_{0}$ within $\pm 30 \%$. This absolute precision is sufficient since often unknown details as attachment to islands may change absolute number densities within that range. The good agreement in $\chi$ and $\eta$ makes both the lattice and the uniform depletion approximation suitable for comparison to experimental island densities for coverages up to saturation. On the other hand, a set of constant capture rates reproduces the correct slope and intercept only for a single coverage, and the geometric concept yields to systematic errors.

The influence of the island shape can also be accounted for by using Eq. (1) with the exponents and prefactors given for the respective cases in Table I. This procedure yields sufficient precision; however, it is only valid around $\theta=0.12$ ML. For coverages below and slightly above, the rate equations have to be integrated, which is a straightforward task in the lattice approximation. ${ }^{62}$ The precision of the analysis of island densities in terms of diffusion parameters within rate theory can reach $\pm 1 \%$ when the approximations used for the capture rates are adapted to the island shape.

\section{LOW-TEMPERATURE EFFECTS}

In addition to the analysis of $i=1$ island densities for $D / F>10^{5}$ in terms of $E_{m}$ and $\nu_{0}$, supplementary information can be inferred from island densities obtained at very low temperatures $\left(D / F \ll 10^{5}\right)$. As mentioned above, the precision of diffusion parameters can be increased significantly by analyzing densities over a larger range of $D / F$, but also details such as attachment to islands and transient mobility play an increasing role for small $D / F$. This is an advantage and a drawback at the same time. It opens up the study of these effects, but it also requires additional experimental information to separate them out.

\section{A. Post-deposition mobility and attachment to islands}

The Arrhenius plot of island densities for $\mathrm{Ag} / \mathrm{Pt}(111)$ down to $D / F=10^{-1}(T=50 \mathrm{~K})$ is shown in Fig. 5. From the experimental data it is seen that there is a linear regime for temperatures from $D / F=4 \times 10^{8}$ down to about $D / F$ $=1 \times 10^{5}$, followed by a downward bending of the island densities measured for lower temperatures. The linear regime reflects the power law expressed in Eq. (1). The downward bending to lower island densities is due to the fact that for lower temperatures, or higher deposition rates, diffusion becomes slow with respect to the incoming flux of adatoms. As a consequence, only a fraction of the deposited adatoms create nuclei or attach to islands during the course of deposition and a considerable monomer density is left after deposition has been terminated. Rapid cooling would preserve these remaining monomers and they would become detectable, for example in STM topographs or in the reflected He intensity. Usually, however, the surface is examined at deposition temperature, and-particularly in the case of STM-also some time after deposition. Therefore, monomers continue to diffuse, leading to island growth and/or nucleation after the desired amount has been deposited. This evolution was labeled post-growth and post-nucleation (in the sense of island growth and nucleation taking place post-deposition). ${ }^{15,35}$

The solid line in Fig. 5 shows the best fit to the experimental data by mean-field rate equations with the self-

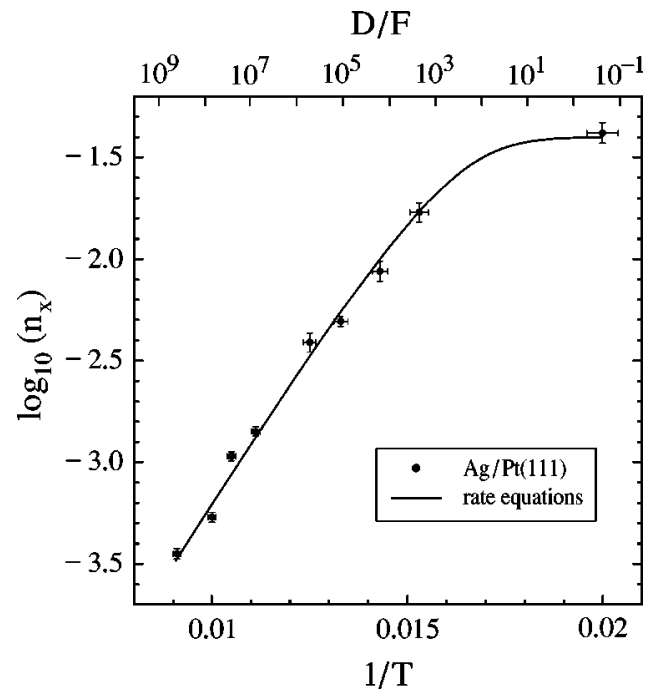

FIG. 5. Comparison of experimental saturation island densities ( $\left.\theta=0.12 \mathrm{ML}, F=1 \times 10^{-3} \mathrm{ML} / \mathrm{s}\right)$ for $\mathrm{Ag} / \mathrm{Pt}(111)$ in the temperature regime where dimers are stable nuclei (and immobile) with self-consistent mean-field calculations for fractal islands. In these calculations the rate Eqs. (2) and (3) have been integrated until $2 \mathrm{~h}$ after deposition in order to account for post-deposition mobility of remaining monomers. Since at very low $T\left(D / F \ll 10^{5}\right)$ not only stable islands but also Ag adatoms appear as immobile protrusions in the STM topographs (Ref. 22), and due to tip convolution these monomers are hardly discernible from islands, the rate equation results show $n_{x}+n_{1}$. This distinction to $n_{x}$ only plays a role for $D / F \ll 10^{5}$.

consistent solutions of the capture numbers of fractal islands. ${ }^{34}$ Taking into account post-deposition mobility, integration of the rate equations was continued until $2 \mathrm{~h}$ after deposition, ${ }^{63}$ the typical time that also elapsed from deposition until STM observation. The mean-field calculation shows excellent agreement with experiment over almost 10 orders of magnitude in $D / F$. It has been performed with a migration barrier of $E_{m}=168 \mathrm{meV}$ and an attempt frequency of $\nu_{0}=7 \times 10^{13} \mathrm{~s}^{-1}$. From a variation of these values we derive a conservative estimation of the overall error (systematic and scattering of data) of $E_{m}=168 \pm 5 \mathrm{meV}$ and $\nu_{0}=7 \times 10^{13 \pm 0.3} \mathrm{~s}^{-1}$. For the interpretation of these diffusion parameters we note that diffusion between adjacent fcc sites on an fcc(111) surface occurs by jumps via hcp sites. Often there is a small binding energy difference between both sites. ${ }^{38,64}$ If there is such a difference for $\mathrm{Ag} /$ $\operatorname{Pt}(111)$, as suggested from theory, ${ }^{65}$ the $E_{m}$ value determined above signifies the rate limiting diffusion process, i.e., the one with the larger barrier. The attempt frequency $\nu_{0}$ stands for jumps into any of the six possible directions. The diffusion constant $D$ is defined in unit cells per second as $D=1 / 3 \nu_{0} \exp \left(-E_{m} / k T\right)$.

To further investigate post-deposition effects and the role of monomer attachment to islands and other monomers we turn now to the analysis of island densities with KMC simulations. The KMC simulations discussed in this paper were done using two independently developed codes, one for a hexagonal lattice ${ }^{66}$ and one for a square lattice. ${ }^{34}$ In Fig. 6, we compare experimental data for the $\mathrm{Ag} / \mathrm{Pt}(111)$ system to KMC simulations that were performed for dendritic islands on a hexagonal lattice and thus accounted for the lattice and 

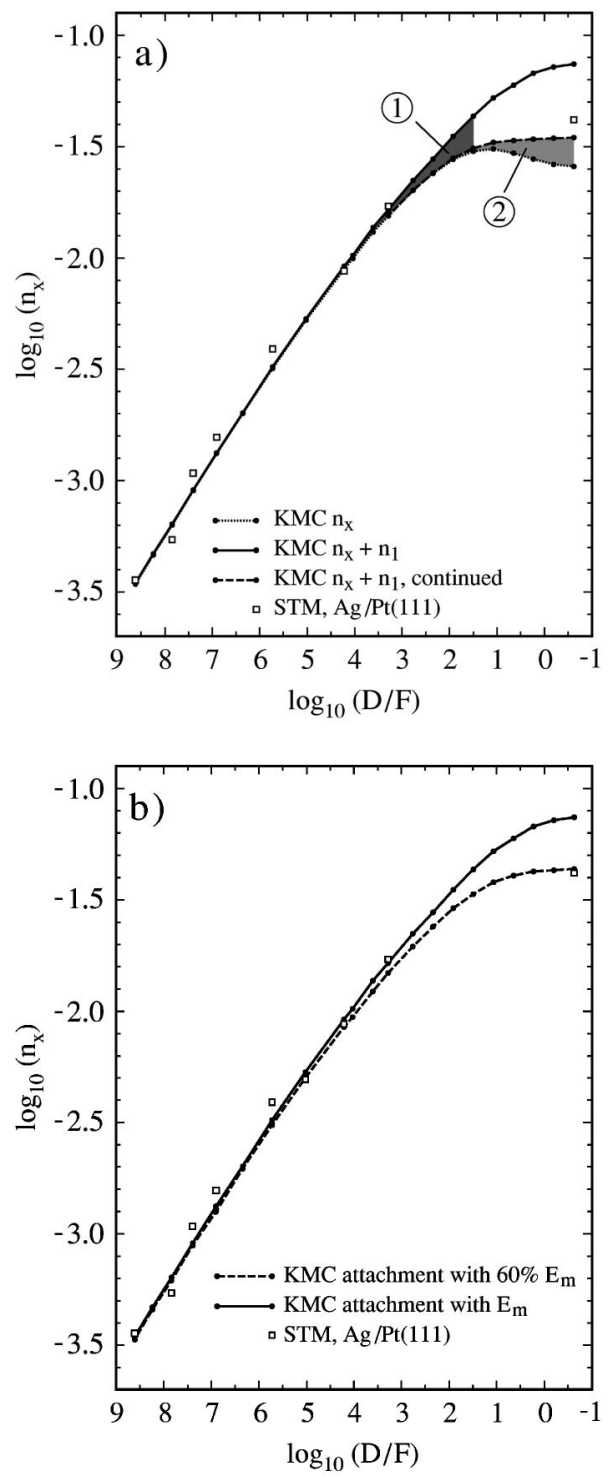

FIG. 6. (a) KMC simulations showing deviations from scaling appearing for $D / F<10^{5}$ and leading to smaller island densities than expected from Eq. (1). The experimental island densities for $\mathrm{Ag} / \mathrm{Pt}(111)$ can be rationalized by post-deposition mobility appearing between deposition and inspection of the surface by STM (notice the effect of counting monomers as islands). (b) KMC simulations with the diffusion parameters for $\mathrm{Ag} / \mathrm{Pt}(111)$ showing the effect of easy attachment to islands. The KMC simulations in (a) and (b) were performed with the parameters $E_{m}=168 \mathrm{meV}$, $E_{\text {corner } \rightarrow \text { A-step }}=160 \mathrm{meV}, E_{\text {coll }}=160 \mathrm{meV}$ for collective dimer relaxation at A-tips (see Ref. 67). A common attempt frequency of $\nu_{0}=7 \times 10^{13} \mathrm{~Hz}$ was used.

island symmetry (see Sec. V.A). To establish comparison with the mean-field analysis above we first address the effect of post-deposition mobility in Fig. 6(a). The simulation results are represented by three curves, displaying $n_{x}$ and $n_{x}$ $+n_{1}$, both directly after deposition, and $n_{x}+n_{1}, 2 \mathrm{~h}$ after deposition. The curves coincide for $D / F>10^{5}$, indicating that post deposition effects are absent or negligible in that range, i.e., diffusion is fast enough that nucleation and growth take place entirely during deposition, and there are only very few monomers left when deposition stops.
For $D / F<10^{5}$, first $n_{x}$ directly after deposition begins to deviate significantly from the scaling behavior expressed in Eq. (1), and second, also the monomers left after deposition reach a detectable amount (see deviation of $n_{x}+n_{1}$ from $n_{x}$ indicated as shaded area 1). Note, however, that $n_{x}$ directly after deposition stays congruent with the curve for $\mathrm{KMC}$ continued until $D / F \approx 1 \times 10^{2}$. This signifies that for $10^{5}$ $>D / F>10^{2}$ the density of stable islands is determined immediately after deposition stops, post-deposition mobility only attaches monomers to existing islands without creating new ones (post-growth). For smaller $D / F, n_{x}$ reaches a maximum at $D / F \approx 2 \times 10^{1}$; afterwards $n_{x}$ decreases towards lower temperatures. This is due to diffusion becoming so slow that only very few islands can be created during deposition and many monomers remain. Accordingly, postdeposition mobility is now responsible for the creation of new islands (post nucleation) raising the curve of $n_{x}$ (see shaded area 2) to yield the characteristic plateau of constant island densities. This plateau is caused by $D$ becoming sufficiently small with respect to $F$ that nucleation takes place almost entirely in the absence of the deposition flux. The diffusion rate, respectively, the substrate temperature, determine the time after deposition that it takes to form all nuclei. Their final density, however, is temperature independent and only a function of coverage, and their size distribution is exponentially decreasing. ${ }^{35}$

The KMC results in Fig. 6(a) for post-deposition mobility correctly describe the experiment for $\mathrm{Ag} / \mathrm{Pt}(111)$ in the whole range of $D / F$. The simulations were performed with identical parameters for monomer diffusion as the analysis within rate theory displayed above in Fig. 5. The agreement of both methods is striking. Here it is demonstrated for KMC simulations performed on a hexagonal lattice with dendritic islands in comparison to rate theory for fractal islands. For a comparison of rate theory with KMC simulations on a square lattice, see Ref. 34.

There is an alternative way besides post-deposition mobility to rationalize the small island densities observed in experiment for $\mathrm{Ag} / \mathrm{Pt}(111)$ for $D / F \ll 10^{5}$. It is attachment of monomers to islands ${ }^{68,69}$ or other monomers ${ }^{70}$ with a lower activation energy than for diffusion on a flat terrace. The KMC results reproduced in Fig. 6(b) demonstrate the effect on island densities when attachment towards islands and other monomers is performed with a reduced barrier of $60 \%$ of the terrace value. This amount is suggested from effective medium theory ${ }^{71}$ calculations for attachment towards twofold coordinated sites for $\mathrm{Ag} / \mathrm{Pt}(111)$ and for $\mathrm{Pt} / \mathrm{Pt}(111){ }^{66}$ The island density detectable in the experiment $\left(n_{1}+n_{x}\right)$ becomes smaller due to the effect of "easy" attachment over one site. The most prominent effect is again located at low temperatures. The results in Fig. 6(b) describe the experiment for $\mathrm{Ag} / \mathrm{Pt}(111)$ quite well without any need for postdeposition mobility. It should be noted that easy attachment is overestimated in the KMC simulations, since there was no distinction between attachment towards onefold and twofold coordinated edge sites, whereas a noticeable reduction of the barrier is inferred from EMT only for attachment to twofold sites.

The available low temperature data for $\mathrm{Ag} / \mathrm{Pt}(111)$ can be explained either by post-deposition effects or by attachment towards islands with a smaller barrier than for terrace 
diffusion. The latter effect is expected to be more pronounced for hexagonal than for square lattices since on the first there is attachment to laterally twofold coordinated sites. A distinction of easy attachment and post-deposition mobility can be achieved by STM measurements where the sample is quenched after deposition freezing post-deposition mobility and preserving monomers eventually remaining after deposition. It is important to notice that post deposition effects and the details of the adsorption potential close to steps (as long as there is no repulsion) are both irrelevant for $D / F>10^{5}$. These effects enter at lower $D / F$ and the data in Figs. 5 and 6(a) and 6(b) show how they can be accounted for.

\section{B. Statistical growth}

Statistical growth is deposition at temperatures where thermally activated diffusion processes are frozen. STM measurements in this temperature regime [see Fig. 7(a)] allow the determination of the mean island size as a quotient of coverage and density. Both numbers are known with sufficient absolute precision that conclusions on transient mobility can be reached. This is achieved by comparison of experiments, monitoring the mean island size as a function of coverage, with theoretical models once with and once without transient mobility. The mean island size expected for pure statistical growth can be estimated with percolation theory existing for square and hexagonal lattices. ${ }^{72}$ Percolation theories neglect deposition onto filled sites, however. Consequently, they yield numbers that are slightly too small compared to epitaxial growth experiments. These effects can be accounted for either by integrating the rate Eqs. (2) and (3), or in KMC simulations. Results from both methods obtained for a hexagonal lattice are displayed in Fig. 7(b), again showing that $\mathrm{KMC}$ is fully consistent with rate theory. The mean island size expected for deposition of $0.1 \mathrm{ML}$ under conditions where atoms stick to their impact site, or roll down from another adatom onto which they were deposited, is 1.48 atoms (percolation theory yields 1.35 atoms). However, if one permits transient mobility over 1 lattice site the expected mean island size is 2.3 atoms [see dashed line in Fig. 7(b)]. The experimental value for the mean island size of $1.2 \pm 0.3$ atoms obtained for $0.1 \mathrm{ML} \mathrm{Ag} \mathrm{deposited} \mathrm{onto}$ $\mathrm{Pt}(111)$ at $35 \mathrm{~K}$ clearly allows one to rule out transient mobility for that system.

Note that easy attachment to islands over one site and transient mobility over one site yield the same mean island size. Transient jumps of condensing atoms increase the mean island size only in those cases where they proceed towards other monomers or islands ending up with attachment. Therefore, the data presented in Fig. 7 also serve to exclude easy attachment with an extremely small barrier, as was observed for $\operatorname{Ir} / \operatorname{Ir}(111){ }^{68}$ Generally, transient mobility can be discerned from easy attachment to islands and monomers as the first is nonthermal whereas the latter is assumed to be thermally activated. Therefore, decreasing the deposition temperature is expected to freeze in easy attachment, whereas transient mobility, if there is any, should persist down to lowest temperatures. Similarly to the present case of $\mathrm{Ag} / \mathrm{Pt}(111)$, transient mobility was also ruled out for $\mathrm{Ni}$ and $\mathrm{Au} / \mathrm{Au}(110)$, based upon comparison between measured and simulated island sizes for low-temperature deposition. ${ }^{73}$ (a) $\mathrm{T}=35 \mathrm{~K}, \Theta=0.1 \mathrm{ML}$
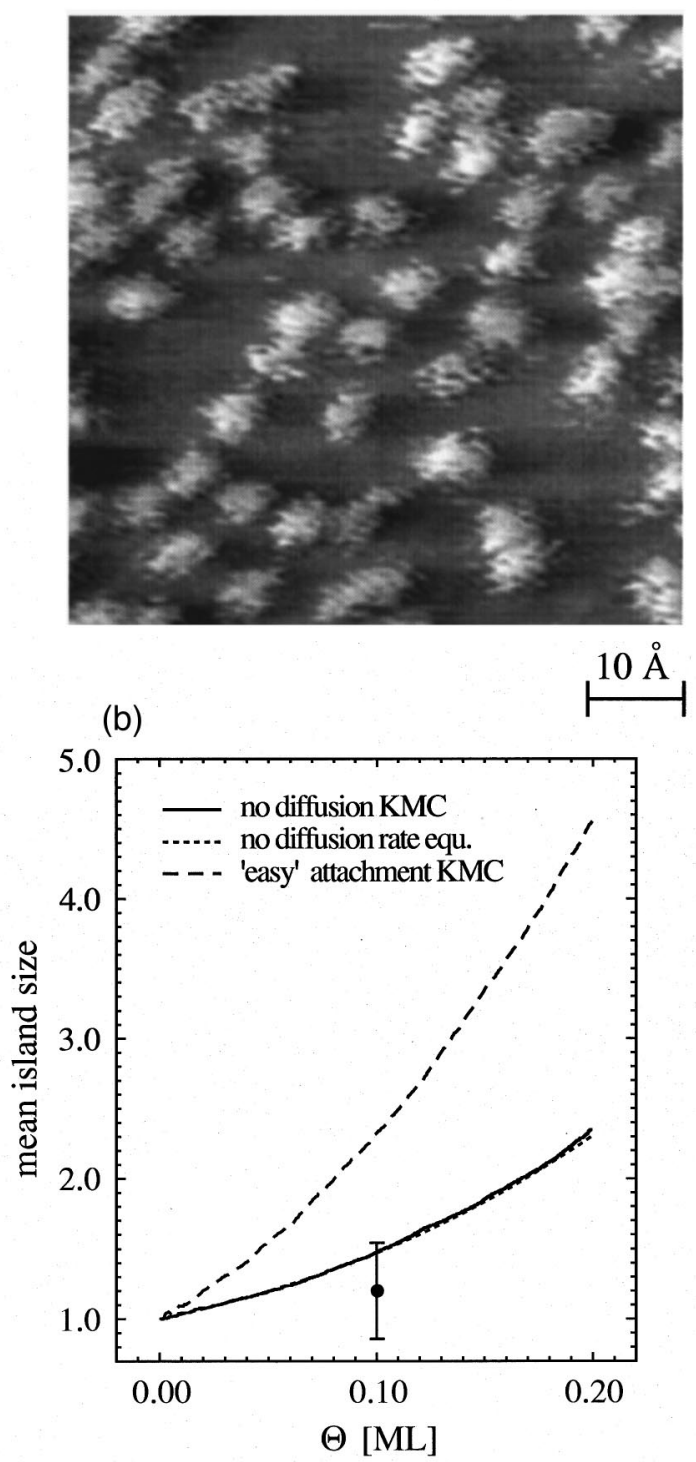

FIG. 7. (a) Deposition of $\mathrm{Ag}$ onto $\mathrm{Pt}(111)$ at $35 \mathrm{~K}$ where terrace diffusion is frozen $\left(D=4 \times 10^{-11}\right.$ unit cells $\left.\mathrm{s}^{-1}\right)$ shows predominantly monomers imaged as bright dots of 4-7 $\AA$ width. Due to tip convolution, monomers are not discerned from dimers. Comparison of the experimental mean island size with the theoretical curves (b) for statistical growth and transient mobility over one site clearly rules out transient mobility for the system $\mathrm{Ag} / \mathrm{Pt}(111)$.

\section{DENSITY SCALING AND COALESCENCE}

In this section, we investigate density scaling for coverages up to coalescence that is particularly important for diffraction techniques requiring a minimum coverage of typically 0.3 ML. We analyze results from kinetic Monte Carlo simulations that establish density scaling for the most common island shapes in the range of $0 \leqslant \theta \leqslant 0.4$ ML.

\section{A. Island shapes}

The islands formed by $\mathrm{Ag}$ on $\mathrm{Pt}(111)$ have dendritic shape as displayed in Fig. 8. Dendritic in this context denotes ramified islands with preferred growth directions ${ }^{74}$ in contrast to DLA clusters, where branches grow in random 
(a) $\mathrm{T}=80 \mathrm{~K}$

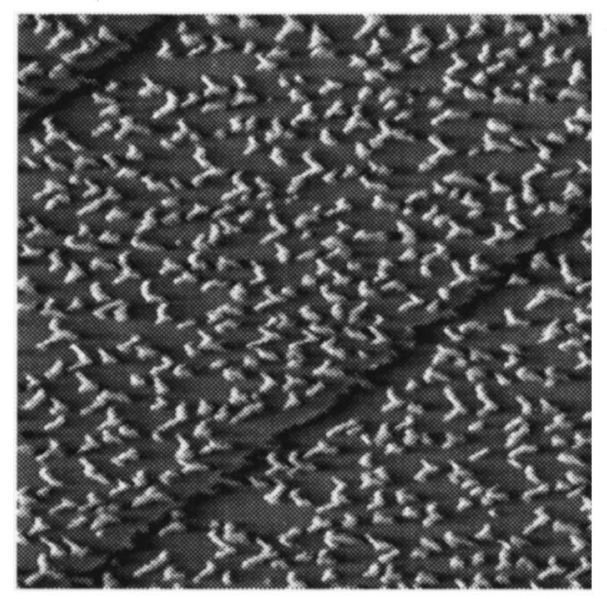

(b) $\mathrm{T}=95 \mathrm{~K}$

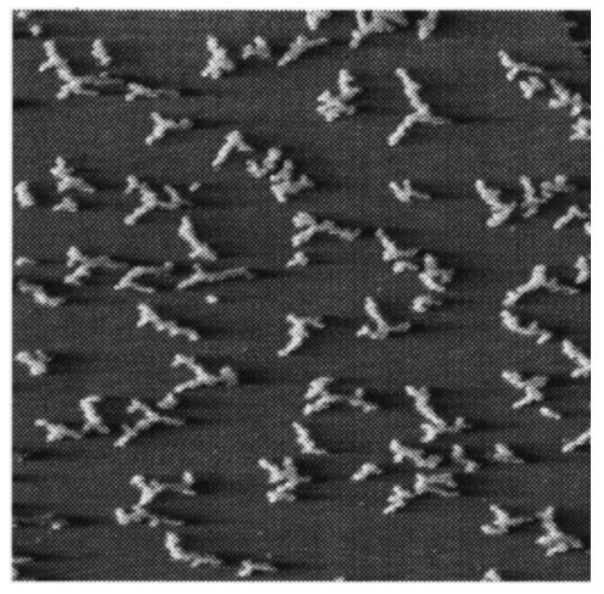

(c) $\mathrm{T}=110 \mathrm{~K}$

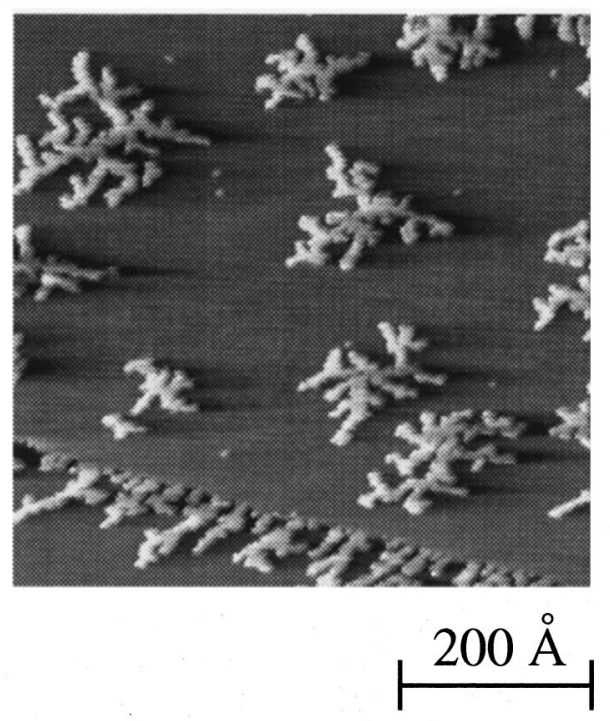

FIG. 8. Variation of the saturation island density with temperature for deposition of $0.12 \mathrm{ML} \mathrm{Ag}$ onto $\mathrm{Pt}(111)$ at 80,95 , and 110 $\mathrm{K}$, respectively $\left(F=1.11 \times 10^{-3} \mathrm{ML} / \mathrm{s}\right)$. See also the evolution of the dendritic island shape with size. The small dendrites at $80 \mathrm{~K}$ are $Y$ 's whereas the large islands at $110 \mathrm{~K}$ begin to branch several times and resemble snowflakes. directions. ${ }^{75}$ The preferential growth directions are the three crystallographic $\langle\overline{11} 2\rangle$ directions, leading to $Y$ shapes for small islands [Figs. 8(a) and 8(b)] while larger islands resemble snowflakes with a triangular envelope [Fig. 8(c)]. The atomic process giving rise to this particular shape is the asymmetry in diffusion of atoms from corner sites (onefold coordinated) towards the two nonequivalent step sites (twofold coordinated) and from terraces at $A$ and $B$ steps, ${ }^{67}$ generally present on a hexagonal surface. ${ }^{77,78}$ For $\mathrm{Ag} / \mathrm{Pt}(111)$, $\mathrm{Ag} / \mathrm{Ag}(111)$, and presumably also for $\mathrm{Pt} / \mathrm{Pt}(111)$ (Ref. 76) corner diffusion towards $A$ steps is activated as soon as terrace diffusion is, since it requires a comparable energy barrier. Theoretical calculations suggest that this is a general trend for close packed surfaces. ${ }^{85}$ This implies that cornerto- $A$-step diffusion is always active when aggregation is and the classical hit-and-stick mechanism yielding DLA clusters does not exist in low-temperature metal epitaxy. In addition to the corner-to-step diffusion asymmetry, there is an asymmetry in the direct attachment to steps always favoring $A$ steps. This leads to asymmetric population of both steps and to the observed branching into preferred directions, as demonstrated by means of KMC simulations. ${ }^{67,76}$ The anisotropy in corner diffusion and attachment generally characterizes close packed substrates, implying that dendritic growth is the rule rather than the exception on these lattices. ${ }^{79,80}$

At higher temperatures (or low flux), the necessary conditions for dendritic growth ( $i=1$ and no dislocations) are no longer fulfilled and branches grow in random directions. ${ }^{81,74}$ Although these islands have larger branches due to edge diffusion, ${ }^{82,83}$ their shape and fractal dimension closely resemble DLA clusters. ${ }^{74,81}$ This renders DLA clusters a relevant island shape, although the classical DLA clusters with monoatomic branches are not expected to form. At even higher temperatures corner crossing becomes activated, finally leading to a transition to compact islands. Therefore, dendritic, DLA clusters (with larger branches) and compact islands can appear on close packed surfaces. On square surfaces there are only laterally onefold coordinated edge sites. This generally implies fast edge diffusion and slightly slower but still fast corner crossing generally leading to compact islands (for an exception, where strain effects are believed to interfere, see Ref. 84).

For the present context of density scaling we investigate the impact of the two types of ramified as well as compact island shapes on the dependence of $n_{x}$ on $D / F$. The specific island shape was accounted for in realistic KMC simulations incorporating the key diffusion processes taking place at the edge. For DLA, edge diffusion was frozen in; for dendrites, diffusion from corner sites to $A$ steps was permitted (the island shapes are exactly those observed in Fig. 8, see caption of Fig. 6 for KMC parameters). For compact islands on a square lattice, the diffusion rate for an edge atom with one lateral bond was set proportional to $F^{1 / 3}$ (see Ref. 83). In the case of compact islands on a hexagonal lattice we have chosen to simulate triangular islands, since this avoids diffusion of smaller clusters (see in more detail below). The simulation results are sumarized in Table II for $\theta=0.12 \mathrm{ML}$. Variations in $\chi$ for the different island shapes are evident. For dendrites $\chi$ is $\frac{1}{3}$ within the statistical error of the simulation. The exponent for DLA islands is increased by $2 \%$, that for compact islands on a square lattice is by $4 \%$ below the classical one, 
TABLE II. Prefactors $\eta$ and slopes $\chi$ for $\theta=0.12$ and $i=1$ [see Eq. (1)] obtained from linear regressions to KMC simulations in the range of $1 \times 10^{5} \leqslant D / F \leqslant 4 \times 10^{8}$ for dendritic, DLA, and compact triangular islands on a hexagonal lattice, and compact islands placed on a square lattice.

\begin{tabular}{lcc}
\hline \hline \multicolumn{1}{c}{ Island shape } & $\eta$ & $3 \times \chi$ \\
\hline Eq. (1) & 0.250 & 1.000 \\
Compact islands, square & $0.23 \pm 0.01$ & $0.96 \pm 0.01$ \\
Compact islands, triangular & $0.20 \pm 0.01$ & $0.94 \pm 0.01$ \\
Dendrites & $0.23 \pm 0.01$ & $0.994 \pm 0.006$ \\
Dendrites easy attachment & $0.21 \pm 0.01$ & $0.982 \pm 0.007$ \\
DLA cluster & $0.23 \pm 0.01$ & $1.016 \pm 0.007$ \\
\hline \hline
\end{tabular}

whereas the scaling exponent for compact triangular islands on a hexagonal lattice is by $6 \%$ below $\frac{1}{3}$. The finding that DLA islands have a larger scaling exponent than compact islands agrees well with earlier results. ${ }^{28,30,34}$ Dendrites are in between the compact and DLA cases, which is expected since they spread out less isotropically and therefore have a smaller variation of the capture cross section with size than DLA clusters.

It becomes obvious upon comparison of Tables I and II that the scaling exponents $\chi$ and the prefactors $\eta$ obtained from KMC agree well with those from rate theory-when performed with the adequate approximations for the capture numbers. Therefore, Monte Carlo simulations and rate theory both yield an equally valid analysis of the experiment.

Attachment to islands via a lower barrier than terrace diffusion reduces the overall island densities by $10 \%$. As pointed out above, easy attachment lowers $n_{x}$ more strongly at small $D / F$; therefore $\chi$ also becomes slightly decreased. Notice that the KMC simulations and the rate theory [see Figs. 5 and 6(a)] both show the slight bending in $\ln \left(n_{x}\right)$ versus $\ln (D / F)$ with respect to the straight line suggested from Eq. (1). This deviation from linear scaling is intrinsic; its magnitude is expressed in the errors given for the $\chi$ values in Tables I and II. The nonlinearity implies that these $\chi$ values are only valid when analyzing island densities in the investigated regime of $1 \times 10^{5} \leqslant D / F \leqslant 1 \times 10^{9}$. Systematic errors of up to twice the error given in Tables I and II arise upon linearly analyzing island densities in only one side of this regime. The prefactor $\eta$ in Eq. (1) giving the absolute number densities is seen from Table II to remain largely unaffected by the island shape.

\section{B. Coalescence and density scaling at larger coverages}

Above, we have analyzed island densities at a constant coverage of $0.12 \mathrm{ML}$ corresponding to the experimental data on to which we based our theoretical discussion. There are several reasons for looking at density scaling also outside the "saturation" regime. Most of the experimental techniques working in reciprocal space require a minimum coverage above saturation. The side bands in diffraction scans obtained with LEED clearly emerge only at and above 0.3 ML. ${ }^{86}$ Also He diffraction experiments were performed at coverages as high as $0.5 \mathrm{ML}^{23}$ To enable a precise analysis of island densities acquired at any coverage we investigate in the present subsection the density scaling over the whole coverage regime up to percolation.
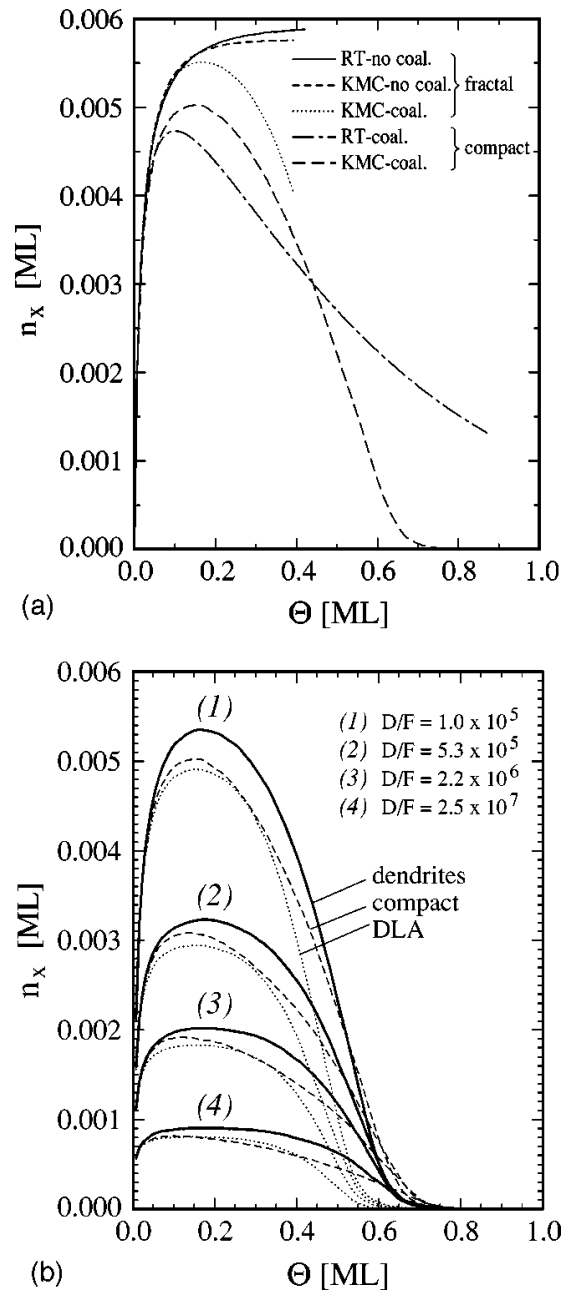

FIG. 9. (a) Coalescence investigated for fractal and compact islands by means of rate theory and KMC simulations on a square lattice performed for $D / F=1 \times 10^{5}$. Coalescence begins to affect island densities at $\theta>0.1 \mathrm{ML}$. Notice the agreement between rate theory and KMC for the curves without coalescence. The coalescence term of mean-field theory overestimates coalescence effects at early times while it does not drop $n_{x}$ to 0 at $\theta=1 \mathrm{ML}$. (b) Island densities for three types of islands that can be realized on a hexagonal lattice. KMC simulations show that coalescence sets in abruptly for dendrites and DLA clusters, whereas compact islands show an extended coalescence regime.

Density scaling for any fixed coverage below saturation can very well be accessed within mean-field nucleation theory (see above). Coalescence, however, is less well described within that theory. The curves for compact islands in Fig. 9(a) show that adding the coalescence term in Eq. (3) yields island densities that are too small at low coverages, whereas coalescence is underestimated at higher coverages. Therefore, the island density does not drop to 0 even long after KMC shows percolation. The failure of nucleation theory in describing coalescence is well known. Spatial correlations between islands become increasingly important once the islands approach each other and there are efforts to improve the coalescence description in mean-field theory. ${ }^{87}$ Also, island size distributions are affected by island correlations and therefore they come out wrong in mean-field theory, too. ${ }^{88}$ These two weak points of mean-field theory are intrinsic and improvement can only evolve by abandon- 
ing the mean-field approach, e.g., by combination with simulation results. The onset of coalescence becomes evident when comparing the curve from KMC simulations neglecting coalescence (coalesced islands were still counted separately) with those taking coalescence into account [fractal island curves in Fig. 9(a)]. Coalescence becomes discernible already at 0.1 ML, but the effects remain small until 0.2 ML; up to this coverage mean-field theory yields reliable results.

The effect of island shape on coalescence is addressed in Fig. 9(b) showing results from KMC simulations performed on a hexagonal lattice for deposition of up to $1 \mathrm{ML}$. For ramified islands it is seen that coalescence sets in suddenly, leading to a steep decrease of the island density, reaching its half maximum at $0.43 \mathrm{ML}$ for DLA, but somewhat later, at $0.48 \mathrm{ML}$, for dendritic growth (these values are for the case of $D / F=10^{5}$ ). The earlier coalescence observed for DLA aggregates is due to the wide and isotropically spread branches of these aggregates, facilitating coalescence. For dendrites coalescence is delayed, since there are only three growth directions. Thinking of the $Y$-shaped dendrites in Figs. 7(a) and 7(b), for coalescence, these branches have to grow towards the center of the neighboring island, which is well screened against random walkers by two of its branches. In contrast to the ramified islands, compact islands start to coalesce early and then the island density slowly diminishes over an extended coverage regime. This difference is due to the fact that for compact islands there is no screening of the narrow spacing between adjacent islands, since the atoms necessary to fill up this space can be supplied by edge diffusion from parts of the edge that are fully exposed to the terrace diffusion field. Note that these general trends for the shape dependence of coalescence are largely independent of $D / F$, respectively, temperature.

Cuts at various coverages through the types of curves for island number densities shown in Fig. 9(b) are represented in an Arrhenius plot in Fig. 10(a). The KMC simulations for dendrites were performed with the diffusion parameters for $\mathrm{Ag} / \mathrm{Pt}(111)$ (see caption Fig. 6 and Ref. 67). It becomes obvious that the island densities obtained for $0.10 \leqslant \theta \leqslant 0.30$ ML all fall into a very narrow regime, whereas the densities at 0.05 and those for $\theta>0.30 \mathrm{ML}$ lie below; finally, the data for $\theta \geqslant 0.50 \mathrm{ML}$ show strong deviations from linearity in $\ln \left(n_{x}\right)$ vs $\ln (D / F)$. For coverages below $0.50 \mathrm{ML}$ the island densities exhibit to a good approximation an exponential law of the form of Eq. (1). The exponent $\chi$ and prefactor $\eta$ obtained from linear regressions to the data in Fig. 10(a) are shown in Fig. 10(b). It is seen that for dendrites the scaling exponent is within $1 \%$ of being identical to the classical one in an extended coverage regime from 0.1 to $0.25 \mathrm{ML}$. Outside this regime the scaling exponent drops by up to $12 \%$ at $0.45 \mathrm{ML}$. This change in scaling is caused by coalescence appearing at slightly different coverages for each $D / F$.

Figure 10(b) also shows results from KMC simulations for DLA clusters on a hexagonal lattice as well as for compact islands on hexagonal and square lattices. The scaling exponent for DLA islands is above that of dendrites until $\theta=0.30 \mathrm{ML}$. At higher coverages $\chi$ drops more rapidly for DLA-clusters than for dendrites due to the earlier coalescence. The scaling exponent for compact islands on a square lattice stays well below $\frac{1}{3}$.
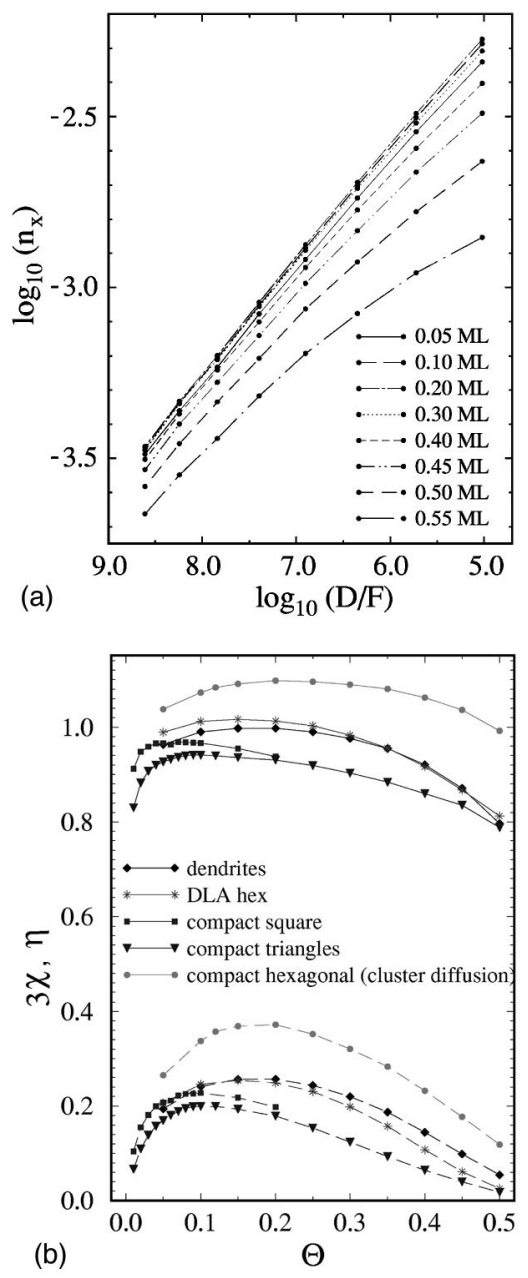

FIG. 10. Density scaling as a function of coverage and island shape as inferred from KMC simulations. (a) Arrhenius plot of island densities for different coverages as obtained from KMC simulations for dendritic islands. (b) The exponent $\chi$ and the prefactor $\eta$ (see full and dashed lines) appearing in the scaling law Eq. (1) as a function of coverage for various island shapes. $\chi$ was scaled such that deviations from the classical value $\chi=\frac{1}{3}$ become apparent. The KMC simulations for dendrites, DLA clusters, and two types of compact islands were performed on a hexagonal lattice. We also show KMC results for compact islands on a square lattice.

As briefly addressed above, a simulation generating compact islands on a hexagonal lattice may involve cluster diffusion. In order to generate hexagonal compact islands, edge diffusion and corner crossing have to be allowed with equal rates for $A$ and $B$ steps. When this is is done, however, small clusters begin to move by edge diffusion. In order to maintain compact hexagonal islands down to $D / F=10^{5}$, the barriers for edge - and by this also cluster - diffusion have to be close to $E_{m}$. Density scaling then becomes perturbed by cluster mobility (see Sec. II B).

We have tried two approaches to addressing compact hexagonal islands in KMC simulations. In the first, we have set for all edge diffusion processes (corner to step, step to step, step to corner) at $A$ and $B$ steps an identical barrier $E_{\text {edge }}$, which was selected such that islands remained compact and hexagonal down to $D / F=10^{5} \quad\left(E_{\text {edge }}=200 \mathrm{meV}\right.$ vs $E_{m}$ $=168 \mathrm{meV}$ ). This simulation shows a scaling exponent up to $10 \%$ above the classical one [see Fig. 10(b)]. This increase 
of $\chi$ is caused by cluster diffusion becoming more rapid at higher temperature where it reduces island densities more strongly. In this first approach, we find that the degree to which $\chi$ increases due to cluster mobility critically depends on $E_{\text {edge }}$ (for $E_{\text {edge }}=180 \mathrm{meV}$ vs $E_{m}=168 \mathrm{meV}$ it reaches $20 \%)$. In a second approach we have varied the common edge diffusion barrier with temperature, such that the edge diffusion processes were allowed with a rate proportional to the lateral impingement rate onto the island edge $I=F / n_{x}$ times the mean edge length defined by $\sqrt{\theta / n_{x}}$. By this we maintained compact hexagonal islands throughout the whole temperature range, while cluster diffusion was less effective at higher temperatures. This moves up the island densities for large $D / F$ and yields scaling exponents by $10 \%$ below the classical one. We note that varying $E_{\text {edge }}$ with $T$ saves computer time in simulations; however, it will hardly be realized in a real system. The simulation results of models 1 and 2 show the degree to which the scaling exponent $\chi$ can become affected by cluster mobility induced by edge diffusion. On the other hand, the results imply that if a real system has compact hexagonal islands with straight steps down to $D / F=10^{5}$, cluster diffusion will play a role in its density scaling.

In order to access density scaling for compact islands on a hexagonal lattice without the complication of cluster diffusion, we have done simulations generating triangular islands on a hexagonal lattice. For this purpose, edge and corner diffusion at $A$ and $B$ steps are discerned, and a common barrier $E_{\text {edge }}$ is associated with some of them (corner to corner at $B$ step, corner to $B$ step, corner to $A$ step and the reverse process, diffusion along $A$ step), while all other processes along the island edge (corner to corner at $A$ step, $B$ step to corner, diffusion along $B$ step) are turned off. This choice of parameters produces compact, triangular islands bound by straight $A$ steps. In these simulations, we find that density scaling is independent of the choice of $E_{\text {edge }}$, as long as it is small enough to generate compact islands throughout the considered scaling regime of $10^{5} \leqslant D / F \leqslant 10^{9}$. The density scaling obtained that way for triangles on a hexagonal lattice almost coincides with the one derived for compact square islands on a square lattice. The shapes of the curves for $\chi$ and $\eta$ represented in Fig. 10(b) reflects the extended coverage regime in which compact islands coalesce. The early onset of coalescence leads to a clear maximum of $\chi$ at $\theta=0.1 \mathrm{ML}$, followed by a rather weak decrease. We mention also that the scaling for compact islands at $\theta \geqslant 0.2 \mathrm{ML}$ generally depends on whether restructuring of two coalesced islands into a single-compact one is permitted. The process required for this is corner breakup. First results indicate that there is continued scaling with smaller variations in $\chi$ when rapid restructuring is allowed.

The results presented in this section enable one to take the effect of island shape into account and to analyze island densities taken at any coverage. This is useful for LEED and He-diffraction studies, but it also enables one to estimate the error when analyzing island densities with the classical values for $\chi$ and $\eta$, as was formerly done. The data represented in Fig. 10(b) allow one to considerably improve this analysis; they extend the applicability of Eq. (1) to a variety of island shapes and to coverages outside the saturation regime.

\section{DISCUSSION AND CONCLUSION}

Our results show that the straightforward analysis of saturation island densities with mean-field nucleation theory by means of Eq. (1), when performed for $D / F>10^{5}$ and a critical nucleus size of $i=1$, allows one to determine the energy barrier and attempt frequency for surface diffusion with an accuracy of $\sim 10 \%$. Increased precision is obtained in the analysis of $n_{x}$ in terms of $E_{m}$ and $\nu_{0}$ when the island shape and lattice symmetry are taken into account. This has been done above in extended KMC simulations that give scaling exponents and prefactors for the principle island shapes that can be realized on hexagonal and square lattices. The island shape effect is to increase $\chi$ by $2 \%$ for DLA islands; it is exactly the classical one for dendrites, whereas $\chi$ is smaller by $4 \%$ for compact islands on a square lattice and by $6 \%$ for compact triangular islands on a hexagonal lattice. Compact hexagonal islands on a hexagonal lattice are a peculiar case in which edge diffusion induces cluster motion with the result of convoluting edge diffusion barriers with $E_{m}$. Cluster diffusion also increases the bending of $\ln \left(n_{x}\right)$ vs $\ln (D / F)$, which makes hexagonal islands escape from simple scaling of the form of Eq. (1). For all other island shapes, the correct values for the coefficients in Eq. (1) are given in Fig. 10(b) as a function of $\theta$, and in Table II for $\theta=0.12 \mathrm{ML}$. This information enables accurate analysis $( \pm 1 \%)$ of total number densities in terms of $E_{m}$ and $\nu_{0}$. That way the error is basically defined by the scatter of the experimental data.

We showed that self-consistent rate theory and KMC simulations are fully consistent with each other. Since both methods require appreciable computational effort, we also showed that some of the classical approximations for the capture numbers yield good agreement with the more costly calculations. The agreement between KMC and rate theory in average quantities establishes application of rate theory for extracting precise parameters of surface diffusion from island densities. For coverages below saturation this analysis can be done by integration of rate equations within approximations for $\sigma$ adequately chosen for the respective island shape. For saturation coverage, analysis in terms of Eq. (1) with the values of $\chi$ and $\eta$ given in Tables I and II suffices. Due to difficulties in the coalescence description of meanfield theory one has to rely on KMC simulations for coverages above saturation.

The island-shape-dependent character of coalescence is reflected in the variations of $\chi$ and $\eta$ with $\theta . \chi$ decreases earlier for both types of compact islands and later but more abruptly for dendrites and DLA clusters. With the information given in Fig. 10(b) it is possible to extend Eq. (1) also to coverages below 0.1 ML and as high as $0.5 \mathrm{ML}$, which should encourage application of the nucleation method also to data from diffraction techniques. The He scattering data for $\mathrm{Cu} / \mathrm{Cu}(100)$ (Ref. 23) were formerly analyzed with a wrong scaling exponent. Redoing this analysis with the proper value for $\chi$ inferred from Fig. 10(b) for compact islands on a square lattice yields $E_{m}=0.34 \pm 0.07 \mathrm{eV}$. This value compares very well to the experimental values published for that system by other authors using different techniques, $E_{m}=0.36 \pm 0.06$ (Ref. 89) and $0.39 \mathrm{eV} .{ }^{90}$

The precision of the analysis of $n_{x}(T)$ data in terms of $E_{m}$ and $\nu_{0}$ of surface diffusion can be enhanced by extension to 
a larger $D / F$ range than the one given by the linear scaling regime. Towards large $D / F$ values the end of the $i=1$ regime sets a natural limit; however, one can extend density measurements towards lower temperature. The results reported above show how information on atomic details, such as attachment to islands via a lower barrier, post-deposition and transient mobility can be gained from island densities collected at low temperatures. Such effects are details which, although appearing similarly at higher temperatures, only affect islands densities at $D / F<10^{5}$. We showed how to account for post-deposition mobility and "easy" attachment, both in mean-field theory and in realistic KMC simulations. This way we obtain $E_{m}=0.168 \pm 0.005 \mathrm{eV} \quad\left(\nu_{0}=7\right.$ $\times 10^{13 \pm 0.3} \mathrm{~s}^{-1}$ ) for the diffusion of $\mathrm{Ag}$ monomers on a $\mathrm{Pt}(111)$ surface upon analyzing $n_{x}$ data in a range of $D / F$ of almost 10 orders of magnitude (compare the theoretical value of $200 \mathrm{meV}$ for that system, Ref. 91).

A similar way to get increased precision on $E_{m}$ and $\nu_{0}$ by extending island density measurements towards lower temperatures was pointed out by Bott et al. ${ }^{8}$ In their nucleation curve method very small amounts $\left(4 \times 10^{-3} \mathrm{ML}\right)$ are deposited at a temperature $T$; subsequently, the substrate is rapidly quenched to very low temperature to freeze in diffusion while characterizing the island density by means of STM. The Arrhenius plot of the island densities shows a linear slope being clearly separated from a regime of a constant "island" density towards low temperatures. This plateau corresponds to statistical growth with immobile monomers as islands. Bott et al. analyzed the slope and the onset temperature of diffusion for $\mathrm{Pt} / \mathrm{Pt}(111)$ by KMC. Their result is $E=0.26 \pm 0.01 \mathrm{eV}\left(\nu_{0}=5 \times 10^{12 \pm 0.5} \mathrm{~s}^{-1}\right)$, again representing a value with remarkable precision. This STM result compares very well to the recent FIM result of $E_{m}=0.260$ $\pm 0.003 \mathrm{eV} .{ }^{38}$ (The former FIM value of $E_{m}=0.25 \mathrm{eV}$ also agrees very well. However, it was based upon measuring the onset temperature of diffusion and assuming a general prefactor..$^{92}$ )

The main differences between measuring a nucleation curve as opposed to the measurement of saturation island densities (nucleation method) are the following. At the onset temperature of monomer diffusion, dimers are normally stable and immobile, hence no additional information on the critical cluster size and cluster mobility are required. Effects of island shape and coalescence are excluded, since the is- lands are small and therefore compact no matter what the details of edge diffusion are, and they are far too small to coalesce. The authors of Ref. 8 also performed measurements of saturation island densities $n_{x}$ for $i=1$, thus enabling a test of their method against nucleation theory. A linear regression to their $\log \left(n_{x}\right)$ vs $1 / T$ data and application of Eq. (1) yields exactly the same result within the error margin $\left(E_{m}=0.26 \mathrm{eV}, \nu_{0}=3 \times 10^{12} \mathrm{~s}^{-1}\right)$ as the one obtained from the nucleation curve. This agreement between nucleation method and the nucleation curve method is due to the fact that (i) deviations from scaling are extremely small for the range of $D / F$ addressed here and (ii) coalescence effects become important only well after $0.1 \mathrm{ML}$. In fact, the method of Bott et al. resembles very closely the nucleation method extended to low $T$ (see, e.g., Fig. 5). As such it represents an alternative way to extract quantitative information on diffusion from nucleation data.

We conclude that care has to be taken when diffusion barriers are extracted from statistical analysis of island number densities. ${ }^{93}$ In experiment one has to worry about $i=1$ and in a few systems also about dimer mobility. In KMC simulations one has to be aware of finite size effects, ${ }^{49}$ the poor quality and limited depth of random number generators, ${ }^{94}$ and one also has to incorporate randomness in time and space. However, if experiments and analysis are carefully done, quite precise values for $E_{m}$ and $\nu_{0}$ can be inferred from island number densities acquired in the $i=1$ regime. The precision of the nucleation method can reach that of careful FIM measurements. Current cross checks between FIM and nucleation method underscore the validity of the latter as a reliable source of diffusion parameters. The nucleation method is applicable to many more epitaxial systems than FIM. It enabled diffusion studies for systems with extremely small diffusion barriers ${ }^{19,95}$ and the mechanism of ordering on dislocation networks could be inferred. ${ }^{18}$ Future subjects that can be addressed via the nucleation method are diffusion and nucleation on surface alloys, or surfactant precovered substrates.

\section{ACKNOWLEDGMENT}

The work by G.S.B. was performed under the auspices of the U.S. Department of Energy by Lawrence Livermore National Laboratory under Contract No. W-7405-Eng-48.
*Permanent addresses: Livermore National Laboratory, Livermore, CA 94551.

†Permanent address: Haldor Topsøe A/S, Nymøllevej 55, DK-2800 Lyngby, Denmark.

${ }^{\ddagger}$ Permanent address: Istituto Nazionale per la Fisica della Materia (INFM) and Centro Fisica delle Superfici e delle Basse Temperature del CNR, Dipartimento di Fisica dell'Università di Genova, via Dodecaneso 33, I-16146 Genova, Italy.

${ }^{1}$ R. Gomer, Prog. Phys. 53, 917 (1990); M. L. Lozano and M. C. Tringides, Europhys. Lett. 30, 537 (1995).

${ }^{2}$ G. Ehrlich, Surf. Sci. 246, 1 (1991); Appl. Phys. A: Solids Surf. 55, 403 (1992).

${ }^{3}$ G. L. Kellogg, Surf. Sci. Rep. 21, 1 (1994).

${ }^{4}$ T. T. Tsong, Atom-Probe Field Ion Microscopy (Cambridge University Press, 1990).
${ }^{5}$ Y. W. Mo, Phys. Rev. Lett. 71, 2923 (1993).

${ }^{6}$ B. S. Swartzentruber, Phys. Rev. Lett. 76, 459 (1996).

${ }^{7}$ T. Zambelli, J. Trost, J. Wintterlin, and G. Ertl, Phys. Rev. Lett. 76, 795 (1996).

${ }^{8}$ M. Bott, M. Hohage, M. Morgenstern, T. Michely, and G. Comsa, Phys. Rev. Lett. 76, 1304 (1996).

${ }^{9}$ T. R. Linderoth, S. Horch, E. Lægsgaard, I. Stensgaard, and F. Besenbacher, Phys. Rev. Lett. 78, 4978 (1997).

${ }^{10}$ M. R. Sørensen, K. W. Jacobsen, and H. Jonsson, Phys. Rev. Lett. 77, 5067 (1996).

${ }^{11}$ J. A. Venables, Philos. Mag. 17, 697 (1973).

${ }^{12}$ J. A. Venables, Phys. Rev. B 36, 4153 (1987).

${ }^{13}$ Y. W. Mo, J. Kleiner, M. B. Webb, and M. G. Lagally, Phys. Rev. Lett. 66, 1998 (1991).

${ }^{14}$ J. A. Stroscio and D. T. Pierce, Phys. Rev. B 49, 8522 (1994). 
${ }^{15}$ H. Brune, H. Röder, C. Boragno, and K. Kern, Phys. Rev. Lett. 73, 1955 (1994).

${ }^{16}$ H. Brune, K. Bromann, H. Röder, K. Kern, J. Jacobsen, P. Stoltze, K. Jacobsen, and J. Nørskov, Phys. Rev. B 52, R14 380 (1995).

${ }^{17}$ J. A. Meyer, J. D. Baikie, E. Kopatzki, and R. J. Behm, Surf. Sci. 365, L647 (1996).

${ }^{18}$ H. Brune, M. Giovannini, K. Bromann, and K. Kern, Nature (London) 394, 451 (1998).

${ }^{19}$ B. Fischer, H. Brune, J. V. Barth, A. Fricke, and K. Kern, Phys. Rev. Lett. 82, 1732 (1999).

${ }^{20}$ M. Bott, T. Michely, and G. Comsa, Surf. Sci. 272, 161 (1992); Rev. Sci. Instrum. 66, 4135 (1995).

${ }^{21}$ H. Röder, H. Brune, J. P. Bucher, and K. Kern, Surf. Sci. 298, 121 (1993)

${ }^{22}$ H. Brune, H. Röder, K. Bromann, and K. Kern, Thin Solid Films 264, 230 (1995).

${ }^{23}$ H. J. Ernst, F. Fabre, and J. Lapujoulade, Phys. Rev. B 46, 1929 (1992).

${ }^{24}$ Q. Jiang and G. C. Wang, Surf. Sci. 324, 357 (1995).

${ }^{25}$ J. W. Evans and M. C. Bartelt, Surf. Sci. 284, L437 (1993).

${ }^{26}$ M. C. Bartelt and J. W. Evans, Surf. Sci. 298, 421 (1993).

${ }^{27}$ J. A. Venables, G. D. T. Spiller, and M. Hanbücken, Rep. Prog. Phys. 47, 399 (1984).

${ }^{28}$ J. Villain, A. Pimpinelli, and D. E. Wolf, J. Phys. (France) 2, 2107 (1992).

${ }^{29}$ J. Villain, A. Pimpinelli, L. Tang, and D. Wolf, J. Phys. (France) 2, 2107 (1992).

${ }^{30}$ C. Ratsch, A. Zangwill, P. Smilauer, and D. D. Vvedensky, Phys. Rev. Lett. 72, 3194 (1994).

${ }^{31}$ M. C. Bartelt, L. S. Perkins, and J. W. Evans, Surf. Sci. 344, L1193 (1995).

${ }^{32}$ J. G. Amar and F. Family, Phys. Rev. Lett. 74, 2066 (1995).

${ }^{33}$ Z. P. Shi, Z. Zhang, A. K. Swan, and J. F. Wendelken, Phys. Rev. Lett. 76, 4927 (1996).

${ }^{34}$ G. S. Bales and D. C. Chrzan, Phys. Rev. B 50, 6057 (1994).

${ }^{35}$ B. Müller, L. Nedelmann, B. Fischer, H. Brune, and K. Kern, Phys. Rev. B 54, 17858 (1996).

${ }^{36}$ S. Liu, L. Bönig, and H. Metiu, Phys. Rev. B 52, 2907 (1995).

${ }^{37}$ M. C. Bartelt, S. Günther, E. Kopatzki, R. J. Behm, and J. W. Evans, Phys. Rev. B 53, 4099 (1996).

${ }^{38}$ K. Kyuno, A. Gölzhäuser, and G. Ehrlich, Surf. Sci. 397, 191 (1998)

${ }^{39}$ G. L. Kellogg and P. J. Feibelman, Phys. Rev. Lett. 64, 3143 (1990)

${ }^{40}$ C. Chen and T. T. Tsong, Phys. Rev. Lett. 64, 3147 (1990).

${ }^{41}$ G. L. Kellogg and A. F. Voter, Phys. Rev. Lett. 67, 622 (1991).

${ }^{42}$ P. J. Feibelman, Phys. Rev. Lett. 58, 2766 (1987).

${ }^{43}$ P. Ruggerone, C. Ratsch, and M. Scheffler, in Growth and Properties of Ultrathin Layers, edited by D. A. King, D. P. Woodruff (Elsevier Science, Amsterdam, 1997), Vol. 8 pp. 490.

${ }^{44}$ G. L. Kellogg, Phys. Rev. Lett. 73, 1833 (1994).

${ }^{45}$ C. M. Zhang, M. C. Bartelt, J. M. Wen, C. J. Jenks, J. W. Evans, and P. A. Thiel, J. Cryst. Growth 174, 851 (1997).

${ }^{46}$ S. C. Wang and G. Ehrlich, Surf. Sci. 239, 301 (1990).

${ }^{47}$ A. Bogicevic, P. Hyldgaard, G. Wahnström, and B. L. Lundqvist, Phys. Rev. Lett. 81, 172 (1998).

${ }^{48}$ M. C. Bartelt and J. W. Evans, Europhys. Lett. 21, 99 (1993).

${ }^{49}$ D. E. Wolf, in Scale Invariance, Interfaces, and Non-Equilibrium Dynamics, edited by M. Droz, K. J. McKane, J. Vannimenus, and D. E. Wolf (Plenum, New York, 1994), p. 1
${ }^{50}$ S. Günther, E. Kopatzki, M. C. Bartelt, J. W. Evans, and R. J. Behm, Phys. Rev. Lett. 73, 553 (1994).

${ }^{51}$ J. W. Evans and M. C. Bartelt, J. Vac. Sci. Technol. A 12, 1800 (1994).

${ }^{52}$ M. C. Bartelt and J. W. Evans, Phys. Rev. B 46, 12675 (1992).

${ }^{53}$ L. H. Tang, J. Phys. (Paris) 13, 935 (1993).

${ }^{54}$ D. D. Chambliss and K. E. Johnson, Phys. Rev. B 50, 5012 (1994).

${ }^{55}$ L. Kuipers and R. E. Palmer, Phys. Rev. B 53, R7646 (1996).

${ }^{56}$ T. R. Linderoth, J. J. Mortensen, K. W. Jacobsen, E. Lægsgaard, I. Stensgaard, and F. Besenbacher, Phys. Rev. Lett. 77, 87 (1996); J. J. Mortensen, T. R. Linderoth, K. W. Jacobsen, E. Lægsgaard, I. Stensgaard, and F. Besenbacher, Surf. Sci. 400, 290 (1998).

${ }^{57}$ S. Stoyanov, Appl. Phys. A: Solids Surf. 50, 349 (1990).

${ }^{58}$ J. A. Venables, J. S. Drucker, M. Krishnamurthy, G. Raynerd, and T. Doust, in Epitaxial Heterostructures, edited by D. W. Shaw et al., MRS Symposium Proceedings No. 198 (Materials Research Society, Pittsburgh, 1990), p. 93.

${ }^{59}$ G. S. Bales, Surf. Sci. 356, L439 (1996).

${ }^{60}$ G. Zinsmeister, Thin Solid Films 7, 51 (1971).

${ }^{61}$ M. J. Stowell, Philos. Mag. 26, 349 (1972).

${ }^{62}$ For the present study, the rate equations were integrated within the various approximations for $\sigma$ using MATHEMATICA.

${ }^{63}$ For this purpose $F$ was defined as time-dependent step function and set to $F=0$ after the deposition time was over.

${ }^{64}$ S. C. Wang and G. Ehrlich, Phys. Rev. Lett. 62, 2297 (1989).

${ }^{65}$ C. Ratsch, A. P. Seitsonen, and M. Scheffler, Phys. Rev. B 55, 6750 (1997).

${ }^{66}$ J. Jacobsen, K. W. Jacobsen, P. Stoltze, and J. K. Nørskov, Phys. Rev. Lett. 74, 2295 (1995).

${ }^{67}$ H. Brune, K. Bromann, J. Jacobsen, K. Jacobsen, P. Stoltze, J. Nørskov, and K. Kern, Surf. Sci. Lett. 349, L115 (1996).

${ }^{68}$ S. C. Wang and G. Ehrlich, Phys. Rev. Lett. 70, 41 (1993); 71, 4174 (1993).

${ }^{69}$ R. Stumpf and M. Scheffler, Phys. Rev. Lett. 72, 254 (1994); M. Villarba and H. Jónsson, Surf. Sci. 317, 15 (1994).

${ }^{70}$ G. Vandoni, C. Félix, R. Monot, J. Buttet, and W. Harbich, Surf. Sci. 320, L63 (1994).

${ }^{71}$ K. W. Jacobsen, J. K. Nørskov, and M. J. Puska, Phys. Rev. B 35, 7423 (1987); P. Stoltze, J. Phys.: Condens. Matter 6, 9495 (1994).

${ }^{72}$ M. F. Sykes and M. Glen, J. Phys. A 9, 87 (1976); M. F. Sykes, D. S. Gaunt, and M. Glen, ibid. 9, 97 (1976).

${ }^{73}$ A. Hitzke, M. B. Hugenschmidt, and R. J. Behm, Surf. Sci. 389, 8 (1997); S. Günther, A. Hitzke, and R. J. Behm, Surf. Rev. Lett. 4, 1103 (1997)

${ }^{74}$ H. Brune, C. Romainczyk, H. Röder, and K. Kern, Nature (London) 369, 469 (1994)

${ }^{75}$ T. A. Witten and L. M. Sander, Phys. Rev. Lett. 47, 1400 (1981).

${ }^{76}$ M. Hohage, M. Bott, M. Morgenstern, Z. Zhang, T. Michely, and G. Comsa, Phys. Rev. Lett. 76, 2366 (1995).

${ }^{77}$ K. Stolt, W. R. Graham, and G. Ehrlich, J. Chem. Phys. 65, 3206 (1976).

${ }^{78}$ T. Michely and G. Comsa, Surf. Sci. 256, 217 (1991).

${ }^{79}$ H. Brune, K. Bromann, K. Kern, J. Jacobsen, K. Jacobsen, P. Stoltze, and J. Nørskov, in Disordered Materials and Interfaces, edied by H. Z. Cummins et al., MRS Symposia Proceedings No. 407 (Materials Research Society, Pittsburgh, 1996), p. 379.

${ }^{80}$ Dendrites have also been observed for $\mathrm{Pt}$ aggregation on $\mathrm{Ru}(0001)$; see F. B. de Mongeot, M. Scherer, B. Gleich, E. 
Kopatzki, and R. J. Behm, Surf. Sci. 411, 249 (1998); and for $\mathrm{Au} / \mathrm{Pd}(111)$, R. J. Behm (private communication).

${ }^{81}$ R. Q. Hwang, J. Schröder, C. Günther, and R. J. Behm, Phys. Rev. Lett. 67, 3279 (1991).

${ }^{82}$ H. Röder, K. Bromann, H. Brune, and K. Kern, Phys. Rev. Lett. 74, 3217 (1995).

${ }^{83}$ G. S. Bales and D. C. Chrzan, Phys. Rev. Lett. 74, 4879 (1995).

${ }^{84}$ B. Müller, L. Nedelmann, B. Fischer, H. Brune, J. V. Barth, and K. Kern, Phys. Rev. Lett. 80, 2642 (1998).

${ }^{85}$ A. Bogicevic, J. Strömquist, and B. I. Lundqvist, Phys. Rev. Lett. 81, 637 (1998).

${ }^{86}$ Q. Jiang, A. Chan, and G. C. Wang, Phys. Rev. B 50, 11116 (1994).
${ }^{87}$ S. Liu, L. Bönig, and H. Metiu, Surf. Sci. 392, L56 (1997).

${ }^{88}$ M. C. Bartelt and J. W. Evans, Phys. Rev. B 54, R17 359 (1996).

${ }^{89}$ H. Dürr, J. F. Wendelken, and J. K. Zuo, Surf. Sci. 328, L527 (1995).

${ }^{90}$ M. Breeman and D. O. Boerma, Surf. Sci. 269/270, 224 (1992).

${ }^{91}$ P. J. Feibelman, Surf. Sci. 313, L801 (1994).

${ }^{92}$ P. J. Feibelman, J. S. Nelson, and G. L. Kellogg, Phys. Rev. B 49, 10548 (1994).

${ }^{93}$ P. J. Feibelman, Phys. Rev. B 52, 12444 (1995).

${ }^{94}$ J. Maddox, Nature (London) 372, 403 (1994).

${ }^{95}$ J. V. Barth, H. Brune, B. Fischer, J. Weckesser, and K. Kern (unpublished). 